\title{
Fundamental Insights into the Covalent Silane Functionalization of NiFe-Layered Double Hydroxides
}

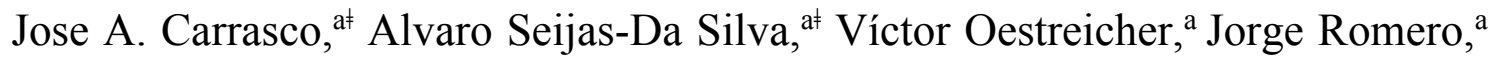 \\ Bence G. Márkus, ${ }^{\mathrm{b}}$ Ferenc Simon, ${ }^{\mathrm{b}}$ Bruno J. C. Vieira, ${ }^{\mathrm{c}}$ João C. Waerenborgh, ${ }^{\mathrm{c}}$ \\ Gonzalo Abellán, ${ }^{\mathrm{a}, \mathrm{d}, *}$ and Eugenio Coronado., ${ }^{\mathrm{a}, *}$
}

\footnotetext{
${ }^{a}$ Instituto de Ciencia Molecular (ICMol), Universidad de Valencia, Catedrático José Beltrán 2, 46980, Paterna, Valencia, Spain.

${ }^{b}$ Department of Physics, Budapest University of Technology and Economics, POBox 91, H-1521 Budapest, Hungary and MTA-BME Lendület Spintronics Research Group (PROSPIN), Budapest, Hungary

${ }^{c}$ Centro de Ciências e Tecnologias Nucleares (C2TN), Instituto Superior Técnico, Universidade de Lisboa, 2695-066 Bobadela LRS, Portugal.

${ }^{d}$ Department of Chemistry and Pharmacy and Joint Institute of Advanced Materials and Processes (ZMP), University Erlangen-Nürnberg, Henkestr. 42, 91054 Erlangen and Dr.-Mack Str. 81, 90762 Fürth, Germany.
}

${ }^{\ddagger}$ These authors contributed equally to this work.

Corresponding authors

Tel.: +49 911 65078-65031. Fax: +49 911 65078-65015, E-mail address: gonzalo.abellan@uv.es (Gonzalo Abellán).

Tel.: +34 96354 4415. Fax: +3496354 3273. E-mail address: eugenio.coronado@uv.es (Eugenio Coronado). 


\begin{abstract}
Layered double hydroxides (LDHs) are a class of 2D anionic materials exhibiting wide chemical versatility and promising applications in different fields ranging from catalysis to energy storage and conversion. However, the covalent chemistry of this kind of 2D materials is still barely explored. Herein, we report the covalent functionalization with silanes of a magnetic NiFe-LDH. The synthetic route consists of a topochemical approach followed by the anion exchange reaction of a surfactant molecules prior to the covalent functionalization with the (3aminopropyl)triethoxysilane (APTES) molecules. The functionalized NiFe-APTES was fully characterized using X-ray diffraction, infrared spectroscopy, electron microscopy, thermogravimetric analysis coupled with mass spectrometry and ${ }^{29} \mathrm{Si}$ solid state nuclear magnetic resonance, among others. The effect on the electronic properties of the functionalized LDH was studied by a magnetic study in combination with Mössbauer spectroscopy. Moreover, the reversibility of the silane-functionalization at basic $\mathrm{pH}$ was demonstrated, and the quality of the resulting LDH proved by studying the electrochemical performance in the oxygen evolution reaction in basic media. Furthermore, the anion exchange capability for the NiFe-APTES was tested employing $\mathrm{Cr}(\mathrm{VI})$, resulting in an increase of $200 \%$ of the anion retention. This report allows a new level in the tunability of LDHs opening the door to the synthesis of new hybrid materials and architectures.
\end{abstract}

\title{
Introduction
}

With the discovery of graphene, ${ }^{[1]}$ the research in two dimensional (2D) materials has gained enormous attention in recent years by the scientific community. ${ }^{[2,3]}$ Indeed, new layered materials have been recently reported such as boron nitride, ${ }^{[4]}$ black phosphorus, ${ }^{[5,6]}$ antimonene ${ }^{[7,8]}$ layered metal chalcogenides, ${ }^{[9,10]}$ or layered coordination polymers, ${ }^{[11]}$ to name a few. In this sort of materials, van der Waals force is the main interlayer interactions that ensembles the crystals. ${ }^{[10]}$ Nevertheless, not only neutral bidimensional materials have been reported, but also ionic ones such as the layered double hydroxides (LDHs). ${ }^{[12]}$ These layered hosts are based in a brucite-like structure composed of cationic sheets consisting of edge shared $\mathrm{M}(\mathrm{OH})_{6}$ octahedra. With a general formula of $\left[\mathrm{M}^{\mathrm{II}}{ }_{1-\mathrm{x}} \mathrm{M}^{\mathrm{III}}{ }_{\mathrm{x}}(\mathrm{OH})_{2}\right]^{\mathrm{x}+}\left(\mathrm{A}^{\mathrm{n}-}\right)_{\mathrm{x} / \mathrm{n}} \cdot \mathrm{mH}_{2} \mathrm{O},{ }^{[13,14]}$ the two constituent metals can vary in a wide stoichiometry range (usually reported between $0.20 \leq \mathrm{x} \leq 0.33$ ) ${ }^{[15]}$ giving rise to a great variety of isostructural layered systems. ${ }^{[15,16]}$ The role of the interlayer anion $\mathrm{A}^{\mathrm{n}-}$ relies in balancing out the excess of positive charge of the layered system. ${ }^{[13,17]}$ One of the most interesting features of this kind of materials is their ability of being exfoliated into 2D charged nanosheets, acting as building blocks to construct more complexes architectures, preserving the main properties of the pristine bulk material. ${ }^{[16,18,19]}$ Another key aspect of LDHs is their unparalleled anion exchange capacity, which allows the substitution of their interlayer anion thanks to anion 
exchange reactions. ${ }^{[20]}$ All this versatility from the point of view of metal composition, stoichiometry, anion exchange, and exfoliation properties position LDHs as excellent candidates for application development in numerous fields of great interest such as catalysis, sensing or magnetism, ${ }^{[16,21]}$ with special importance in the attention-growing energy storage and conversion field. ${ }^{[22-27]}$

Interestingly, one of the less explored aspects within the chemistry of LDHs is covalent functionalization. During the last years, ${ }^{[28-30]}$ covalent functionalization of $2 \mathrm{D}$ materials has been extensively employed due to the following key aspects: (i) improved processability via the reinforcement of the interfacial interactions between material and substrate, ${ }^{[28]}$ (ii) the enhancement of the dispersibility of the samples ${ }^{[31]}$ and (iii) better control of molecular orientation. ${ }^{[32]}$ LDHs are not exempt in this topic, and some reports were published in recent years ${ }^{[33,34]}$ using silanes as anchoring molecules to achieve the covalent binding to the cationic sheets of the LDH material in much the same way as it has been done in other clay materials such as montmorillonite or hydrotalcite. ${ }^{[35-38]}$ Due to the ionic nature of the LDHs, covalent grafting remains a challenging yet attractive strategy in order to widen the scope of action of these clay materials by adding new functionalities. Among the different LDH compositions, NiFe-LDH stands out as one of the best materials in electrocatalysis, and more concretely in the oxygen evolution reaction (OER). ${ }^{[24,25,39,40,27]}$ In addition, its magnetic behaviour at low temperatures ${ }^{[15,24]}$ adds interest for the study of its properties after the functionalization. In this work, we report for the first time the covalent functionalization of NiFe-LDH with (3-aminopropy)ltriethoxisilane (APTES), a complete physical and structural characterization, as well as a thorough magnetic study combined with Mössbauer spectroscopy in order to understand how the covalent functionalization affect the overall structure of this layered material. ${ }^{[29,30]}$ In the light of the results, we demonstrate that grafting of the silane molecules induce several modifications in the chemistry of LDHs such as an enhancement of the adsorptive properties towards contaminant anions, while leaving its magnetic properties barely affected. Moreover, we proved that this covalent functionalization is completely reversible at basic $\mathrm{pH}$ keeping intact the electrochemical performance. This covalent functionalization adds a new level of functionality and complexity to magnetic and electrocatalytic LDHs, paving the way for the design of more complex hybrid architectures.

\section{Results and Discussion}

The synthesis of the NiFe-APTES (Figure 1A and S1) was achieved after successive anion exchange reactions starting from the pristine anthraquinone-intercalated $\mathrm{NiFe}-\mathrm{LDH}(\mathrm{NiFe}-\mathrm{AQ})$ material synthesized via topochemical approach. ${ }^{[20,41,42]}$ The choice for this topochemical 
approach, instead of the most typical hydrothermal assisted synthesis, is motivated by the good crystallinity and the possibility to carry out additional anion exchange reactions to obtain the final material due to its large basal space of $c a .18$ Å. In stark contrast, the hydrothermal approach usually yields carbonate/nitrate-intercalated LDHs, being time-consuming towards the exchange reactions ${ }^{[43,44]}$ In order to allow the insertion of APTES moiety and induce the covalent grafting, a previous enlargement of the basal space is mandatory. For this purpose, the incorporation of a more labile DS molecules (DS-intercalated LDH) by anion exchange reaction is carried out prior to the final inclusion of APTES (Scheme 1). ${ }^{[27]}$ In addition, it is demonstrated that the organophilicity increases after the $\mathrm{DS}^{-}$intercalation, ${ }^{[45]}$ hence favouring the insertion of the smaller APTES molecules into the interlayer space and their condensation with the $-\mathrm{OH}$ groups found on the LDH surface. The last step consists of the grafting of the APTES molecules at $50{ }^{\circ} \mathrm{C}$ under reflux conditions to a previously dried NiFe-DS using DCM as a solvent to avoid the presence of water.

The successful synthesis of the APTES-intercalated material was characterized by different techniques. XRPD analysis (Figure 1B) confirmed the LDH phase of the whole NiFe-LDH family highlighting the main three characteristic basal reflections (003), (006) and (009) in each sample. ${ }^{[15]}$ The (003) peak is related with the basal space of the LDH, therefore shifting to lower 2-theta values as the length of the interlamellar anion increases. ${ }^{[46,47]}$ Thanks to the excellent crystallinity exhibited, the diffraction patterns for the -AQ and -DS samples have been indexed and refined by assuming a hexagonal lattice with $R 3 m$ rhombohedral symmetry. The basal spaces were found to be $c a$. 20, and $22 \AA$ for the-AQ, and-DS samples, respectively, in good agreement with that reported in the literature. ${ }^{[23,33,41,48]}$ With respect to the-APTES sample, on a first glimpse we observed a reduction in the basal space to ca. $18 \AA$ (taking into account that the (003) peak appears at $4,77^{\circ}$ ) with respect to the -DS material.. ${ }^{[48]}$ In addition, the ubiquitous presence of the characteristic (110) peak, proper to the intralayer distance, at around $60^{\circ}$ in the whole family is indicative for the preservation of the LDH phase after the anion exchange and functionalization reactions. ${ }^{[20,49]}$

For the functionalized material, it is important to point out the appearance of great a number of basal reflections as well as an initial strong peak at $c a .2^{\circ}$ that it is usually omitted in most of the diffractograms reported in the literature for silylated-LDH materials. This pattern may be indicative of a second-staging phenomenon in the NiFe-APTES sample between the bilayer arrangement of DS molecules and the APTES molecules, leading to a interstratified compound (Figure S1) ${ }^{[50-53]}$ As explained by He et al.,${ }^{[35]}$ when DS is found in the reaction media (as it is prior the insertion of APTES), it can induce a sharp reflection at low angles ( $c a .2 .7^{\circ}$ ) that is assigned to a new basal reflection, and suggestive of the coexistence of DS anions entering within the interlayer space of the LDH into a bilayer disposition and APTES molecules. ${ }^{[42]}$ Indeed, as 
mentioned by Meyn et al., for a carbon number of 12 as found in the DS anion, a basal spacing between $c a .23 \AA$ and $40 \AA$ can be observed for the single and double layer orientation within the LDH layers, respectively. In our case one can find the basal reflections related with the APTES molecules, the DS molecules as well as the ones originated by the combination of both. To shed light in the possibility of having a staging phenomenon and discard the physical mixture of two different phases, we have selected the different peaks and calculated the associated d-spacing value, followed by the estimation of the corresponding basal space taking into account the $(00 l)$ indexation of the peaks, and assuming a lower intensity for the DS (003) peak, as previously observed by He and co-workers (Figure S2 and Table S1) ${ }^{[35]}$ By doing that we can tentatively identify the peaks related with the APTES molecules and the ones with the DS molecules. However, the first reflection appearing at $2^{\circ}$ does not individually belong to any phase yet its basal spacing matches perfectly with the combination of both interlayer anions (Figure 2 and Table S1). This fact may suggest the presence of a second-staging phenomenon over the existence of a mixture of the two different phases (see inset in Figure S2).

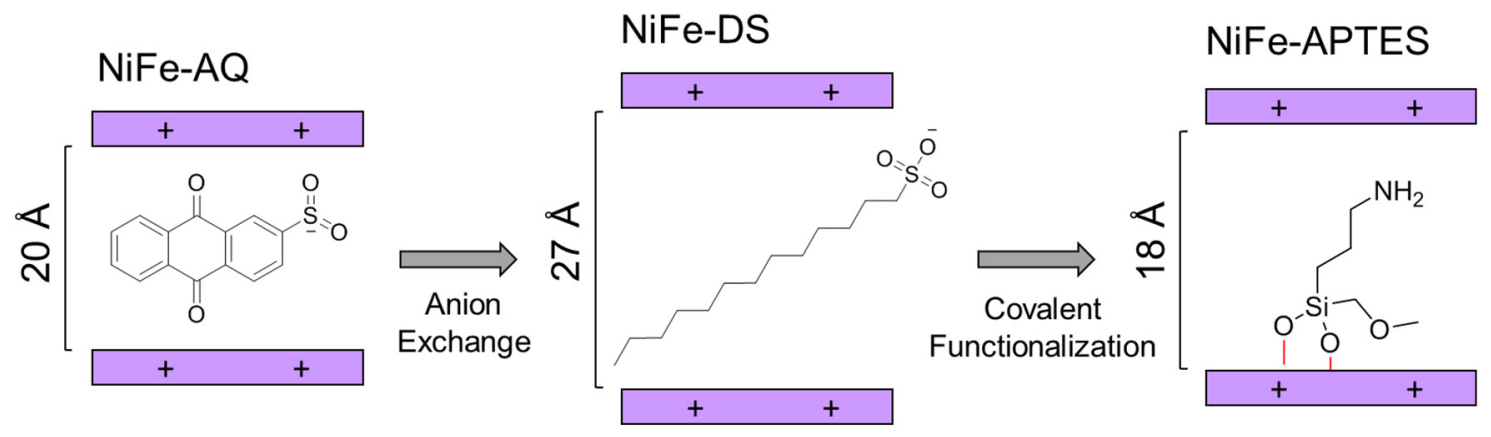

Scheme 1. Set of reactions needed to synthesize the NiFe-APTES sample via the topochemical approach. Purple rectangles indicate the positive layers of the NiFe-LDH. Red bonds refer to the presence of covalent bonding between the APTES molecules and the LDH layer.

The attenuated total reflectance Fourier-transform infrared spectroscopy (ATR-FTIR) of the family also depicts the presence of the APTES molecules in the LDH structure (Figure 1C). According to NiFe-APTES sample, the bands at $c a .3300 \mathrm{~cm}^{-1}$ are related with the presence of $\mathrm{O}-\mathrm{H}$ stretching vibrations from both the hydroxyl group of the LDH or absorbed water molecules. This broad band overlaps with the asymmetric and symmetric stretching modes of the amino groups in the APTES molecules. ${ }^{[54,33,35]}$ The band found at $c a .3000 \mathrm{~cm}^{-1}$ is ascribed to the $\mathrm{C}-\mathrm{H}$ stretching vibrations in the molecules. ${ }^{[55]}$ Comparing with the NiFe-DS sample, it is noticeable its higher intensity of the $\mathrm{CH}$ groups due to the presence of the surfactant moiety with a larger amount of $\mathrm{CH}$ groups. The diminishing in the intensity of the bands related with the surfactant anion after the insertion of the APTES molecules suggests the predominant replacement of the dodecylsulphate molecules, in good agreement with that observed in the XRPD. In addition, a N- 
$\mathrm{H}$ band is observed in the APTES molecules at $c a .1630 \mathrm{~cm}^{-1}$. At lower wavenumbers, the bands in the $1100-1000 \mathrm{~cm}^{-1}$ region are related to additional $\mathrm{Si}-\mathrm{O}-\mathrm{Si}$ and $\mathrm{Si}-\mathrm{O}-\mathrm{C}$ bands. ${ }^{[56-60]}$ The description of the FTIR spectra for the NiFe-DS and NiFe-AQ samples can be found elsewhere, pointing out their main vibrational bands. ${ }^{[41,61]}$ In line with that, to gain further insights into the anion displacements, a porosity analysis was carried out for the NiFe-LDH samples (Figure S3). As pointed out in the $\mathrm{N}_{2}$ isotherms at $77 \mathrm{~K}$, all samples exhibited a type IV behaviour, in good agreement with the expected for these kind of materials. ${ }^{[62-64]}$ While the initial NiFe-AQ displays a Brunauer-Emmett-Teller (BET) surface area of $c a .60 \mathrm{~m}^{2} \cdot \mathrm{g}^{-1}$, this value abruptly decreases down to $11 \mathrm{~m}^{2} \cdot \mathrm{g}^{-1}$ (close to the threshold for non-porous materials measured in our equipment) after the exchange of the DS moieties. ${ }^{[65]}$ However, once the APTES molecules have replaced the DS ones, the BET surface area exhibits an increase up to $c a .19 \mathrm{~m}^{2} \cdot \mathrm{g}^{-1}$. As previously pointed out by the XRPD and FTIR, this change regarding the DS-intermediate is indicative of the successful insertion of the APTES molecules.

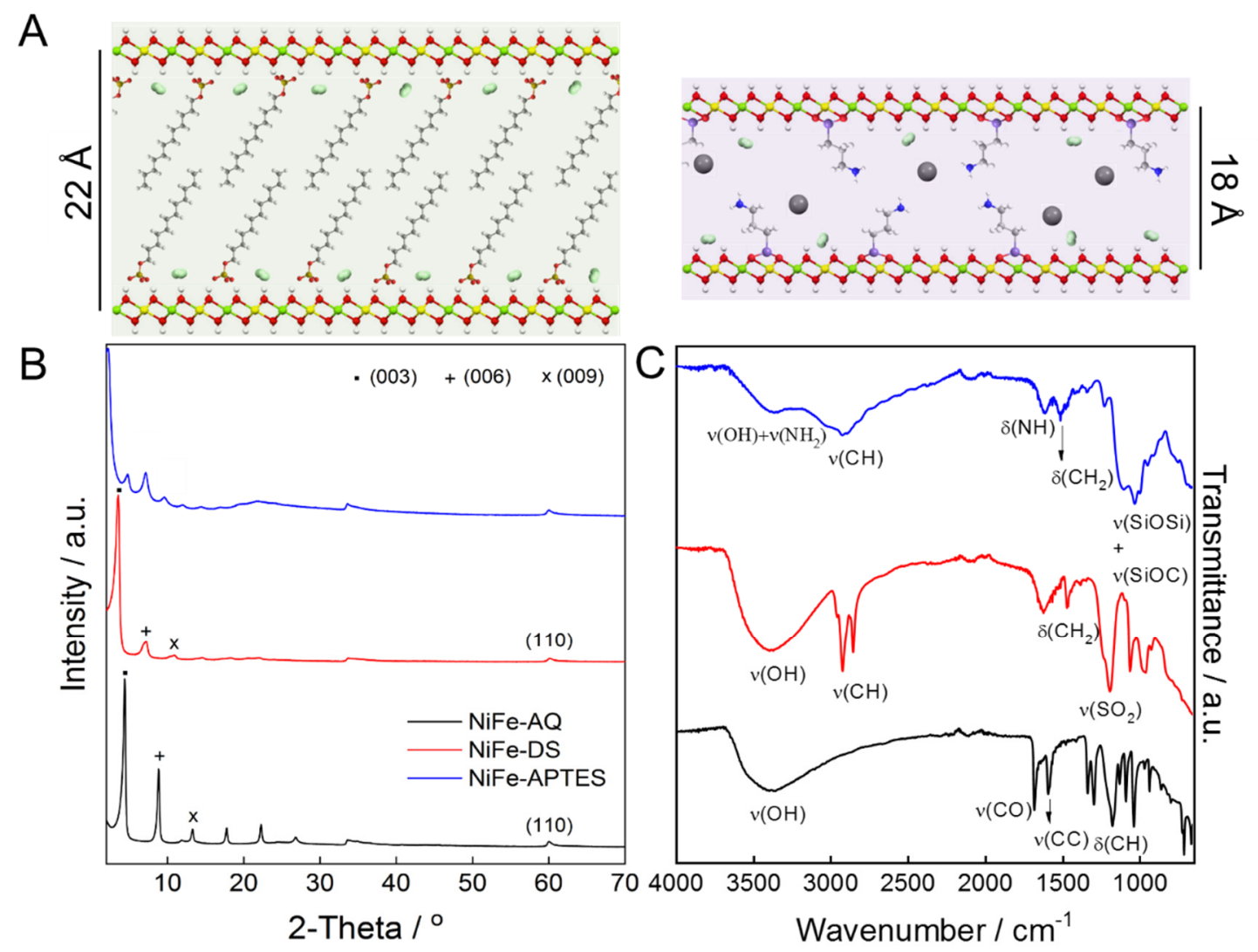

Figure 1. (A) Idealized scheme of NiFe-APTES LDH with the two phases that conforms the secondstaging interstratified compound as shown in Figure S1: (left) bilayer arrangement of DS molecules and (right) with APTES functionalization. (B) XRPD diffractograms and (C) FTIR spectra of the NiFe-LDH family, with their most intense reflections and bands labelled. NiFe-AQ (black line), NiFe-DS (red line) and NiFe-APTES (blue line). The complete discussion of the NiFe-APTES XRPD can be found in Figure S2 and Table S1. 
The morphology and composition of the functionalized material was studied by different microscopies and spectroscopies. Field-emission scanning electron microscopy (FESEM) of $\mathrm{NiFe}$-APTES exhibits an irregular morphology composed of agglomerates, displaying flexible LDH flakes which gather themselves into bigger aggregates of several nanometers that reminds of a flower-like morphology as reported in literature (Fig 3A). ${ }^{[62,66-68]}$ High-resolution transmission electron microscopy (HRTEM) allows a better definition of the flexible LDH flakes (Fig 3B), and the selected area electron diffraction (SAED) pattern suggests different orientations, typically reported for this kind of polycrystalline samples. ${ }^{[62]}$ Dynamic light scattering (DLS) (inset in Fig 3A) measurements in solution depict an average hydrodynamic size in the range of 0.5 to $1 \mu \mathrm{m}$, in good agreement with the results of FESEM and TEM, suggesting the presence of these agglomerates in suspension. EDS confirms the presence of $\mathrm{Ni}, \mathrm{Fe}, \mathrm{Si}, \mathrm{Br}$ and $\mathrm{Cl}$ in the functionalized sample. Moreover, a mapping analysis of the NiFe-APTES carried out in the TEM (Figure 3) highlights the homogeneous distribution of $\mathrm{Ni}, \mathrm{Fe}, \mathrm{Si}, \mathrm{Cl}, \mathrm{Br}$ in these aggregates, with the ratio $\mathrm{Ni}: \mathrm{Fe}=1.9$ and $\mathrm{Si}: \mathrm{Fe}=2.3$. In addition to this, an additional mapping analysis was performed at lower magnification in SEM (see Figure S4).

Furthermore, SEM, TEM and DLS over the NiFe-AQ and NiFe-DS were performed (see, Figure S5-S6), exhibiting the same flower-like morphology found for the functionalized sample. EDS analysis depict an average Ni:Fe ratio of 2.1 and 2.0 for NiFe-AQ and NiFe-DS, respectively, in good agreement with that expected experimentally. Mapping analysis of $\mathrm{NiFe}-$ AQ and NiFe-DS samples are found in Figure S4 indicating a homogeneous distribution for both $\mathrm{Ni}$ and Fe metals throughout the whole samples. 

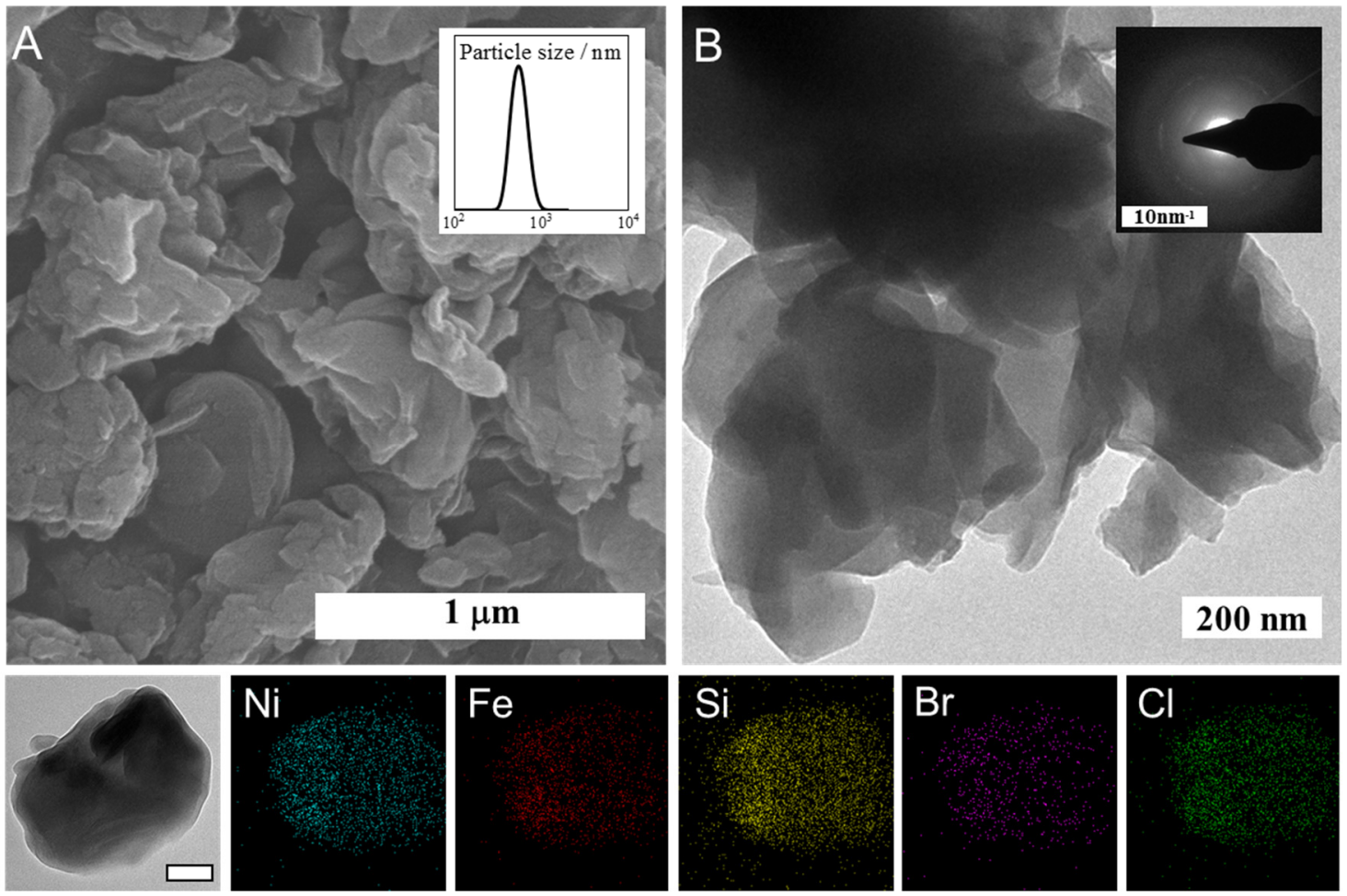

Figure 3. FESEM (A), TEM (B) and mapping images (bottom panel) of NiFe-APTES. The inset in (A) shows DLS and the inset in (B) shows the SAED pattern. Mapping images are obtained from the single particle in the bottom left image (scale bar of $100 \mathrm{~nm}$ ).

Since this topochemical approach gives rise to an irregular flower-like morphology for all the synthesized samples, we have further explored the silane functionalization over a well-defined hexagonal CoAl-LDH obtained through homogeneous precipitation. The main objective relies on checking the possibility of grafting an APTES molecules to a different LDH composition, confirming the homogeneity in the metallic distribution on a micrometric particle. For the synthesis of the CoAl-LDH family we carried out an initial homogeneous coprecipitation approach, followed by successive anion exchange reactions to obtain the final CoAl-APTES material. This can be easily achieved given that $\mathrm{Al}^{3+}$-containing LDH are favoured towards anion exchange reactions (see supplementary information with the experimental details). ${ }^{[13,20]}$ Figure S7 and S8 confirm the successful grafting of the APTES molecules in the LDH, pointing out the hexagonal morphology and good crystallinity of the resulting CoAl-APTES. In line with that, mapping analysis confirmed the homogeneous distribution of $\mathrm{Co}, \mathrm{Al}$ and $\mathrm{Si}$ throughout the whole sample. Therefore, the successful covalent grafting of different LDH compositions synthesized by distinct synthetic approaches, i.e., the topochemically-synthesized $\mathrm{NiFe}-$ and coprecipitationsynthesized $\mathrm{CoAl}-$ here reported and $\mathrm{ZnCr}-\mathrm{LDH}$ reported in literature, ${ }^{[33]}$ proves the general application of the functionalization step. These experiments corroborate that the functionalization strategy is independent of the LDH synthetic methodology, the morphology of the particles and the chemical composition. 
XPS spectroscopy was performed on the NiFe-APTES in order to gain a deeper degree of characterization in terms of chemical speciation and composition on the surface of the materials (Figure 4). Survey plot (Figure 4A) confirms the presence of the main elements $\mathrm{Ni}, \mathrm{Fe}, \mathrm{Si}, \mathrm{Br}$, $\mathrm{Cl}, \mathrm{O}, \mathrm{N}$ and $\mathrm{C}$, in concordance with EDS and mapping analysis. In terms of the metallic ratio, XPS confirms the expected 2:1 ratio, in good agreement with the observed by EDS (Table 1). The presence of $\mathrm{Ni}^{\mathrm{II}}$ was supported by its main peaks at $855 \mathrm{eV}\left(\mathrm{Ni} 2 \mathrm{p}_{3 / 2}\right)$ and $873 \mathrm{eV}\left(\mathrm{Ni} 2 \mathrm{p}_{1 / 2}\right)$, and their satellite peaks at 861 and $879 \mathrm{eV}$, respectively (Figure 4B). ${ }^{[69]}$ At the same time, Fe ${ }^{\mathrm{III}}$ was confirmed according to its two peaks at $711 \mathrm{eV}$ and $725 \mathrm{eV}$, related with the $\mathrm{Fe} 2 \mathrm{p}_{3 / 2}$ and $\mathrm{Fe}$ $2 p_{1 / 2}$ signals, respectively (Figure 4C) ${ }^{[69]}$ Regarding the APTES grafting, Si $2 p$ spectrum depicted a peak centred at $102 \mathrm{eV}$ ascribed to $\mathrm{Si}-\mathrm{O}$ bonding (Figure 4D). ${ }^{[70]}$ The high ratio $\mathrm{Si}: \mathrm{Fe}=9$ suggest the presence of silane polymerization (i.e. monodentate or bidentate grafted molecules, vide infra) on the surface of the LDH as detected by the XPS, in stark contrast with the ratio Si:Fe $=2.3$ obtained via the EDS measurement that encompass the whole LDH structure. $\mathrm{N} 1 \mathrm{~s}$ spectrum (Figure 4E) suggests the presence of three types of nitrogen's environments with contribution of $-\mathrm{NH}_{2}(c a .399 \mathrm{eV}),-\mathrm{NH}_{3}{ }^{+}(c a .401 \mathrm{eV})$ and $-\mathrm{NH}_{3}{ }^{+} \mathrm{SiO}^{-}(c a .403 \mathrm{eV})$, where the $-\mathrm{NH}_{3}{ }^{+}$group represents more than $65 \% \cdot{ }^{[71]}$ Finally, $\mathrm{C}$ 1s spectrum (Figure 4F) notices about the presence of at least three kinds of environments: $\mathrm{C}-\mathrm{C}(c a .284 .5 \mathrm{eV}), \mathrm{C}-\mathrm{N}(c a .286 \mathrm{eV})$ and $\mathrm{C}=\mathrm{O}(c a .284 .5$ $\mathrm{eV})$, which can mostly be attributed to a small contamination of carbonate anions. ${ }^{[71]}$
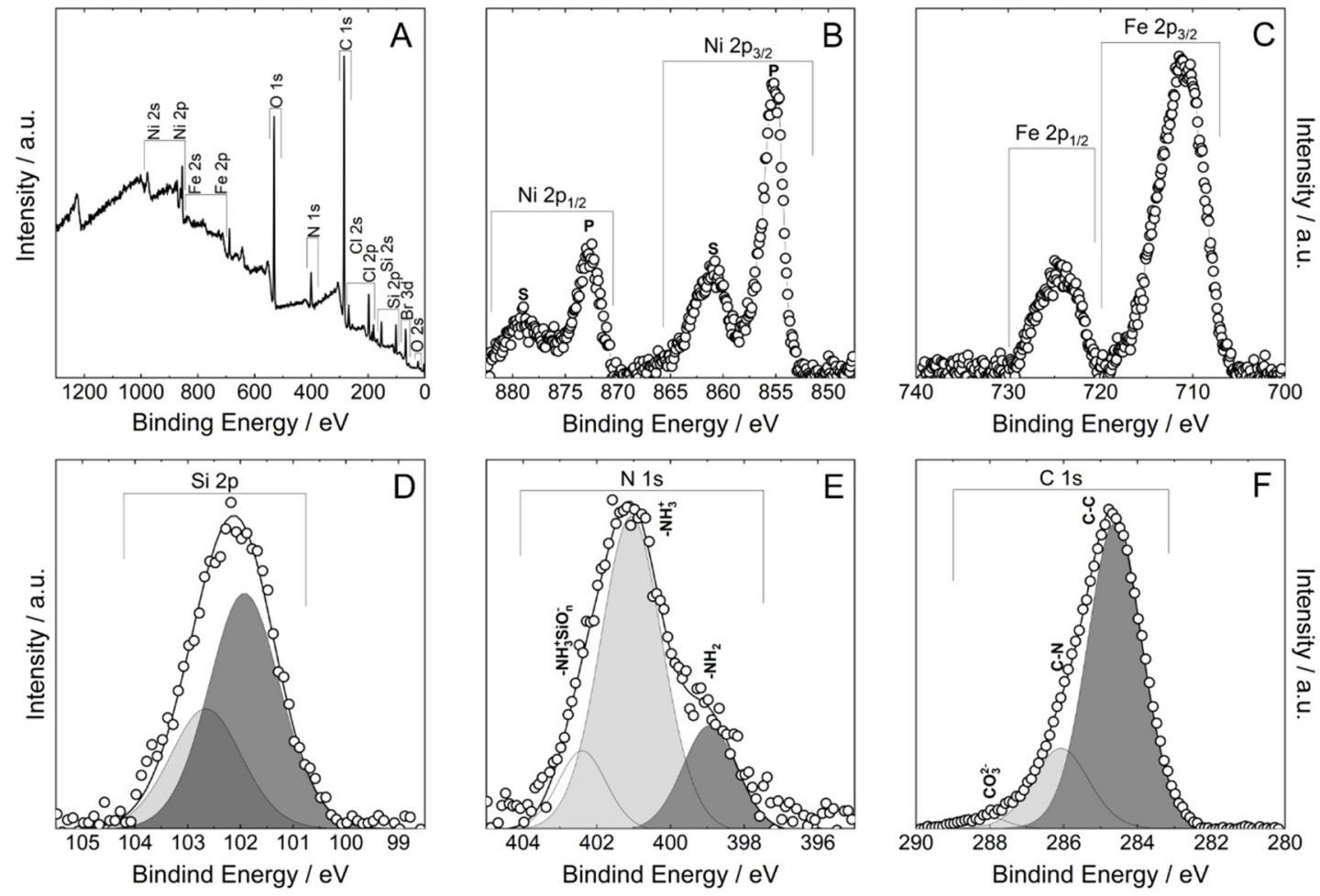

Figure 4. XPS plots for the NiFe-APTES LDH sample. Survey plot highlighting the main analysed elements (A), Ni 2p (B), Fe 2p (C), Si 2p (D), N 1s (E), C 1s (F) spectrums. 


\begin{tabular}{cc}
\hline \multicolumn{2}{c}{ Atomic ratio } \\
\hline $\mathrm{Ni}: \mathrm{Fe}$ & 2.0 \\
$\mathrm{Si}: \mathrm{Fe}$ & 8.9 \\
$\mathrm{Si}: \mathrm{N}$ & 1.1 \\
\hline
\end{tabular}

Table 1. Atomic ratios extracted from the XPS analysis.

In addition to the XPS measurements, further evidence of the covalent functionalization of $\mathrm{NiFe}-\mathrm{APTES}$ can be provided by static NMR and TG-MS measurements. As previously reported in literature, solid state ${ }^{29} \mathrm{Si}$ NMR can be used to characterize the nature of a covalent bonding via a silane molecules, as well as its grafting mode in function of the concept of teeth (dent). ${ }^{[72,73]}$ The ${ }^{29} \mathrm{Si}$ NMR measurement was recorded over the NiFe-APTES sample, depicting a broad structure with a total width of more than a thousand ppm. The relaxation time is found to be isotropic among the peaks. The spin-lattice relaxation time $\left(T_{1}\right)$ is shorter than $100 \mathrm{~ms}$, and the spin-spin relaxation time $\left(T_{2}\right)$ is shorter than $100 \mu \mathrm{s}$. The broadening is caused by the strong dipole-dipole interaction of the Si nuclei, indicating that the APTES molecules are covalently bonded through oxo(hydroxyl) bridges to the hydrotalcite layer. Furthermore, the lines are also broadened due to the dipole-dipole interaction from the near lying magnetic nuclei. ${ }^{[74]}$ Moreover, Fe and $\mathrm{Ni}$ are rich in electrons, whose magnetism also affects the Si nuclei and results in the significant line broadening. The deconvolution of the signal employing three Lorentzian curves are denoted as $\mathrm{T}^{1}, \mathrm{~T}^{2}$ and $\mathrm{T}^{3}$, following the notation of $\mathrm{Q}$. Tao et al. ${ }^{[75]}$ The deconvoluted curves can be associated with three different siloxane bond attached to the layers, related with a monodentate, bidentate or tridentate grafting (Figure 5). In the light of the calculated results, the monodentate and bidentate grafting modes are the main ones, with a relative area of $54 \%$ and $40 \%$, respectively (Table 2 ). The tridentate grafting only depicts a contribution of $6 \%$. Despite of the broadening, which hinders the exact underlying geometry; the parameters of the curves are useful to estimate to distribution of the siloxane bond in the sample. 


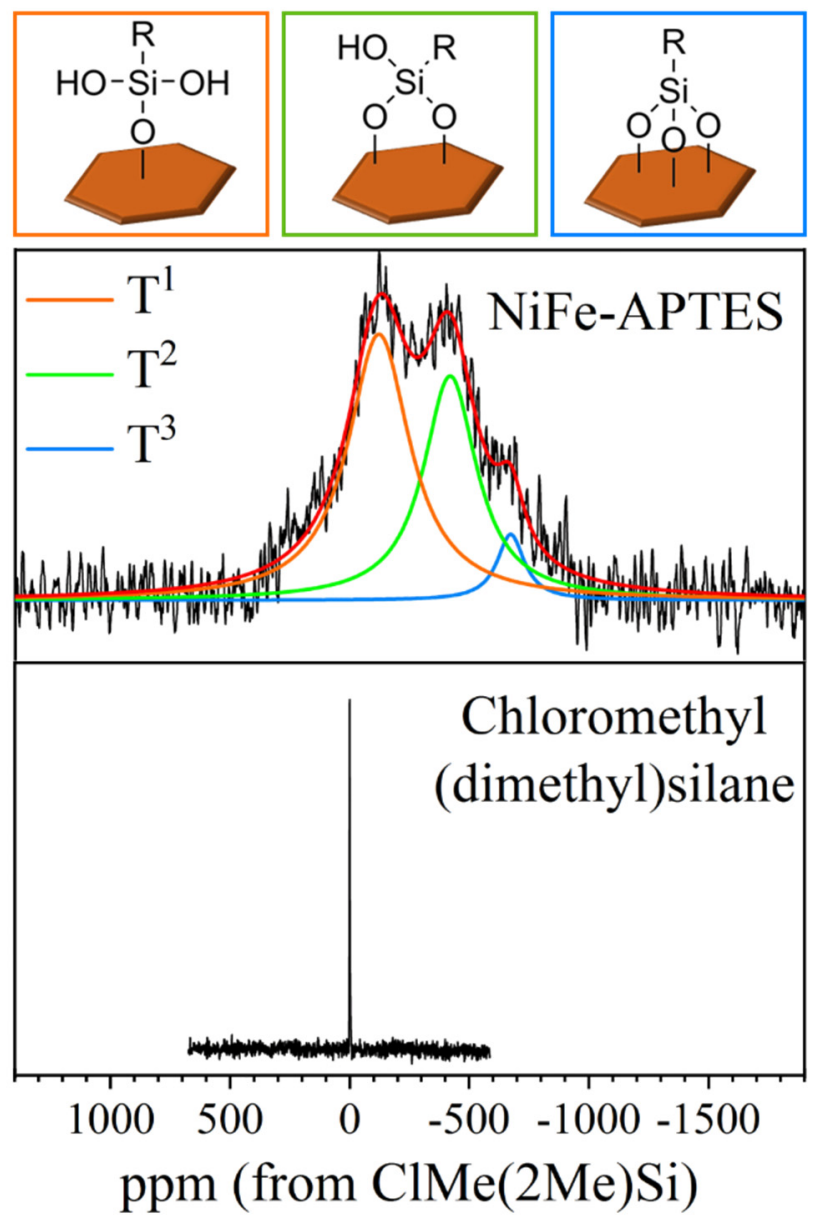

Figure 5. ${ }^{29} \mathrm{Si} \mathrm{NMR}$ of the NiFe-APTES sample, highlighting the different bondings of the APTES molecules to the LDH layer: monodentate $\left(\mathrm{T}^{1}\right.$, orange line), bidentate $\left(\mathrm{T}^{2}\right.$, green line) or tridentate $\left(\mathrm{T}^{3}\right.$, blue line) (top). Chloromethyl(dimethyl)silane was used as reference (bottom spectrum).

\begin{tabular}{ccccc}
\hline Sample & $\begin{array}{c}\text { Chemical shift } \\
\text { from reference } \\
\mathbf{( p p m )}\end{array}$ & $\begin{array}{c}\text { Width } \\
\mathbf{( p p m )}\end{array}$ & $\begin{array}{c}\text { Relative area } \\
\mathbf{( \% )}\end{array}$ & Assignation \\
\hline \multirow{2}{*}{ NiFe-APTES } & $-121 \pm 2$ & $314 \pm 6$ & $54 \pm 1$ & $\mathrm{~T}^{1}$ : monodentate \\
& $-419 \pm 2$ & $270 \pm 9$ & $40 \pm 2$ & $\mathrm{~T}^{2}$ : bidentate \\
& $-672 \pm 3$ & $137 \pm 13$ & $6 \pm 1$ & $\mathrm{~T}^{3}$ : tridentate
\end{tabular}

Table 2. Parameters extracted from the ${ }^{29} \mathrm{Si}$ NMR curves of the NiFe-APTES LDH sample.

In addition to the ${ }^{29} \mathrm{Si} \mathrm{NMR}$, a thermogravimetric analysis coupled with a mass spectrometer (TG-MS) analysis was carried out under an inert atmosphere of helium to provide additional information related to the chemical composition and thermal stability of the functionalized NiFeAPTES sample (Figure 6). As previously stated in the literature, TG-MS is a powerful technique that can be used to characterize both solid and liquid materials. Thanks to the careful 
monitorization of the mass sample and the analysis of the gases that leave the material when heated, this technique allows gathering additional information on the sample composition in base of its constituents. ${ }^{[76]}$ The aim of this technique is focused on the determination of the stability of the APTES molecules attached to the inorganic layers as well as the identification of the intercalated moieties within the LDH structure. ${ }^{[24,77]}$ Typically, the TG profile of a LDH material consists of a first weight loss in the $25-220^{\circ} \mathrm{C}$ range, related with the elimination of the physisorbed water molecules by the LDH structure (solvation molecules). ${ }^{[78]}$ The following and most intense weight loss is the one observed in the $220-600{ }^{\circ} \mathrm{C}$ range, which matches with the overlapping of different processes such as the dehydroxylation of the layered system, the elimination of the chemisorbed water and of the interlayer anion. ${ }^{[66]}$ Afterwards, one can expect a final step above $600{ }^{\circ} \mathrm{C}$ where the crystallization of the resulting oxides occurs. ${ }^{[24]}$ In the present case, the combination of these coupled techniques allows an unequivocal interpretation of different steps during the TG experiment. As a first step, at $c a .95^{\circ} \mathrm{C}$ the loss of $\mathrm{H}_{2} \mathrm{O}$ molecules $(\mathrm{m} / \mathrm{z} 18)$ is observed, related to the physisorbed ones. Furthermore, a more intense signal is observed at $c a .185^{\circ} \mathrm{C}$, which can be attributed to the loss of interlayer $\mathrm{H}_{2} \mathrm{O}$ molecules. By last, there is an additional signal at $c a .310{ }^{\circ} \mathrm{C}$ related with the loss of $\mathrm{H}_{2} \mathrm{O}$ molecules, in this case assigned to the decomposition/dehydroxylation process of the LDH structure. At the same time, the collapse of the LDH structure is accompanied by the loss of the functionalization, evidenced by the signal at $\mathrm{m} / \mathrm{z} 58$ ascribed to the fragment $-\mathrm{CH}_{2} \mathrm{CH}_{2} \mathrm{CH}_{2} \mathrm{NH}_{2}$ of the amino-molecules. Regarding the loss of the inorganic intercalated anions, it takes place at higher temperatures. At ca. $435^{\circ} \mathrm{C}$ there are the signals related to chlorine and bromine anions in the form of $\mathrm{Cl}_{2}(\mathrm{~m} / \mathrm{z} 72)$ and $\mathrm{Br}(m / z 79)$. In addition, the typical TG analysis for NiFe-AQ and NiFe-DS samples can be found at Figure S9, exhibiting the expected two-region profile with an initial loss in the 25-220 ${ }^{\circ} \mathrm{C}$ range and the final dehydroxilation of the layers in the $220-600{ }^{\circ} \mathrm{C}$ region. 


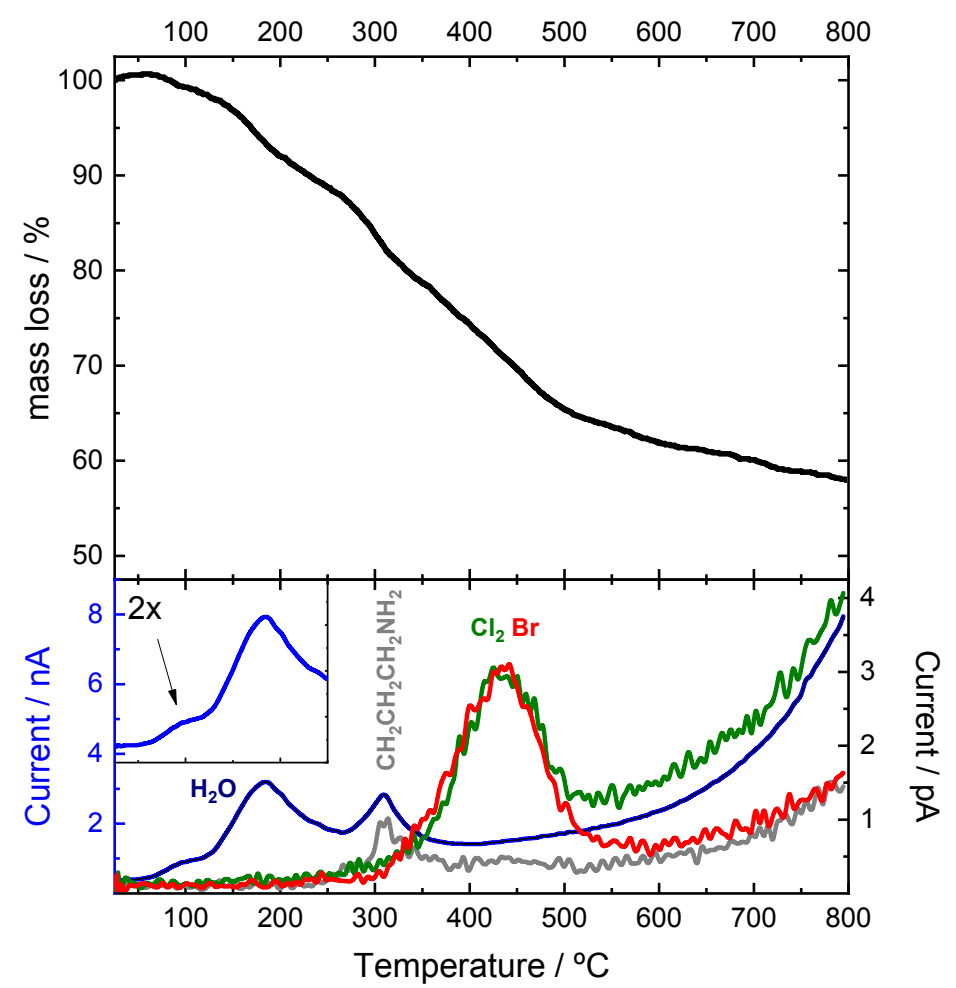

Figure 6. TG-MS analysis of the NiFe-APTES sample, highlighting the different steps in function of the monitored component. The mass traces correspond to $\mathrm{H}_{2} \mathrm{O}(\mathrm{m} / \mathrm{z} 18), \mathrm{CH}_{2} \mathrm{CH}_{2} \mathrm{CH}_{2} \mathrm{NH}_{2}(\mathrm{~m} / \mathrm{z} 58), \mathrm{Cl}_{2}$ $(\mathrm{m} / \mathrm{z} 72)$ and $\mathrm{Br} \cdot(\mathrm{m} / \mathrm{z} 79)$.

Gathering all the data extracted from EDS, elemental analysis, XPS and TG-MS we can estimate the molecular formula for each compound, whose molecular weight reveals crucial for analysing the magnetic data since they are represented per mole of a compound. Molecular formulas are depicted in Table 3 for the NiFe-APTES and Table S2 for the -AQ and -DS intermediate samples. It is worth noting to point out the difficulty in the estimation of the molecular formula for the-APTES intercalated LDHs due to the high number of their constituents and here we report for the first time a complete formula for a functionalized LDH by combining the aforementioned techniques. Comparing both-DS and-APTES results we can observe the expected reduction of the DS molecules being replaced by new APTES molecules.

\begin{tabular}{ccccccc}
\hline Molecular formula $^{a}$ & \multicolumn{3}{c}{ EA found (EA calculated) } & \multicolumn{2}{c}{ EDS } \\
& $\mathbf{C}$ & $\mathbf{H}$ & $\mathbf{N}$ & $\mathbf{S}$ & Ni:Fe & Si:Fe \\
\hline$\left[\mathrm{Ni}_{0.66}{ }^{2+} \mathrm{Fe}_{0.34}{ }^{3+}(\mathrm{OH})_{2}\left(\mathrm{C}_{3} \mathrm{H}_{8} \mathrm{NSi}\right)_{0.28}\left(\mathrm{C}_{3} \mathrm{H}_{9} \mathrm{NSi}\right)_{0.52}\right]^{0.86+}$ & 15.3 & 4.7 & 5.3 & 0.13 & \multirow{2}{*}{1.9} & \multirow{2}{*}{2.3} \\
$\mathrm{Cl}_{0.67} \mathrm{Br}_{0.18}\left(\mathrm{C}_{12} \mathrm{H}_{25} \mathrm{SO}_{4}\right)_{0.009} 0.5 \mathrm{H}_{2} \mathrm{O}$ & $(14.3)$ & $(4.6)$ & $(5.3)$ & $(0.13)$ & &
\end{tabular}

Table 3. Estimated molecular formula for the NiFe-APTES LDH sample.

${ }^{a}$ Molecular formula was estimated from the EDS microanalysis alongside with TG-MS, elemental analysis and XPS. Estimated molecular weight for $\mathrm{NiFe}-\mathrm{APTES}=210 \mathrm{~g} \cdot \mathrm{mol}^{-1}$. 
The magnetic behaviour of the whole LDH family was characterized to study the possible influence of the covalent grafting on the magnetic properties of the LDH. First, it is worth mention that the overall magnetism of a LDH system is controlled by two main contributions: the intralayer magnetic superexchange interactions which occur between metallic centres through the hydroxyl bridges $(-\mathrm{OH})$ across the cationic sheets, and the less intense interlayer dipolar interactions that take place between different layers. ${ }^{[79]}$ Whereas LDHs such as CoAl-LDHs usually behave as ferromagnets, ${ }^{[80]}$ the NiFe-LDHs show a more complex magnetic scenario, as result of the coexistence of ferromagnetic superexchange $\mathrm{Ni}-\mathrm{OH}-\mathrm{Ni}$ interactions with $\mathrm{Ni}-\mathrm{OH}-\mathrm{Fe}$ and $\mathrm{Fe}-$ $\mathrm{OH}-\mathrm{Fe}$ antiferromagnetic superexchange contributions. ${ }^{[79]}$ Moreover, for high Fe concentrations such as the ones depicted here (Ni:Fe ratio of $c a$. 2:1) we can expect significant Fe clustering (AF $\mathrm{Fe}-\mathrm{OH}-\mathrm{Fe}$ pairs) leading to the presence of spin frustration. ${ }^{[15]}$ All magnetic measurements were carried out in a SQUID with freshly prepared powdered samples. Main magnetic data for the whole NiFe-LDH family is depicted in Table 4, in addition to the magnetic data of a typically hydrothermal-synthesized $\mathrm{NiFe}-\mathrm{CO}_{3} \mathrm{LDH}$ for the sake of comparison. ${ }^{[24]}$ The magnetic characterization graphs of the NiFe-AQ and NiFe-DS samples can be found in Figure $\mathbf{S 1 0}$ and

Figure S11, respectively. It is worth mentioning that all magnetic measurements were carried out in eicosane, since this diamagnetic material allows a better immobilization of these small anisotropic crystals, precluding any artefact in the magnetic measurements.

DC susceptibility measurements for all samples exhibit spontaneous magnetization at low temperatures. $\chi_{\mathrm{M}}$ depicts a sharp increase near $c a .20 \mathrm{~K}$ after keeping nearly constant during the cool-down process, reaching a maximum value at $c a .2 \mathrm{~K}$, which is indicative of cooperative magnetic interactions. Attending to the thermal variation of $\chi_{\mathrm{M}} \cdot \mathrm{T}$, there is a smooth decrease from the signal at room temperature to a broad minimum at $c a .50 \mathrm{~K}$, followed by a sharp increase with a maximum signal at $c a .12 \mathrm{~K}$ prior to an abrupt decrease. According to the magnetic measurements, no contaminant magnetic phases were observed for any member of the NiFe-LDH family, since extrinsic magnetic impurities such as Fe-spinels or oxides exhibit a temperaturedependent component. ${ }^{[81]}$ By comparing all samples, the NiFe-APTES exhibits lower magnetic signal than the other ones, which can be related with the lesser metallic contribution to the total molecular weight of the NiFe-APTES than for the other samples. By fitting the inverse of the magnetic susceptibility $\left(\chi_{M}{ }^{-1}\right)$ to a Curie-Weiss law in the high temperature regime (50-300 K) we can estimate a positive Weiss constant $(\theta=9.5 \mathrm{~K})$, indicative of the predominance of ferromagnetic interactions in the system (Figure 7A). In the light of the results, both $\mathrm{NiFe}-\mathrm{CO}_{3}$ $\mathrm{HT}$ and $\mathrm{NiFe}-\mathrm{APTES}$ samples exhibit positive values of $\theta$, related with the predominance of ferromagnetic interactions, while $\mathrm{NiFe}-\mathrm{AQ}$ and $\mathrm{NiFe}-\mathrm{DS}$ samples display negative values of $\theta$, i.e., predominance of antiferromagnetic interactions. Previous studies have demonstrated that no direct and simple predictions can be done connecting the increase in the basal space with the 
nature of the Weiss constant. ${ }^{[20,47,82]} \mathrm{FC} / \mathrm{ZFC}$ highlighted the cooperative magnetism of the samples at low temperatures. From the point where FC and ZFC diverges we can estimate a blocking temperature of the system (Figure 7B). For our as-synthesized LDHs, both NiFe-DS and NiFe-APTES displayed values of $c a .13 \mathrm{~K}$, somewhat lower than for the other materials. A lower value of these ordering temperatures may be ascribed to the presence of size effects. ${ }^{[24]}$ To obtain a more accurate study of the ordering temperature, we performed $\mathrm{AC}$ dynamic susceptibility measurements in the presence of an external field of 3.95 Oe oscillating at 1,10 , 110, 333 and $997 \mathrm{~Hz}$ (Fig 7D). Both the in-phase ( $\left.\chi^{\prime}{ }_{\mathrm{M}}\right)$ and the out-of-phase ( $\left.\chi^{\prime \prime}{ }_{\mathrm{M}}\right)$ signals exhibited a defined peak at low temperatures, confirming the cooperative magnetism in the sample. From the out-of-phase signal we can extract the temperature for the onset of the spontaneous magnetization $\left(\mathrm{T}_{\mathrm{M}}\right)$, defined as the point where $\chi_{\mathrm{M}} \neq 0$ and can be used as an accurate value for the magnetic ordering of the system. The estimated $\mathrm{T}_{\mathrm{M}}$ value for the NiFeAPTES $\left(\mathrm{T}_{\mathrm{M}}=15.7 \mathrm{~K}\right)$ is within the range of the values obtained for other NiFe-LDHs ( $c a .16-$ $22 \mathrm{~K}$ ) (Figure 7D). ${ }^{[15]}$ Hysteresis cycle recorded at $2 \mathrm{~K}$ exhibited spontaneous magnetization at low temperatures, with a coercive field higher than 1000 Oe in all cases, in good agreement with other NiFe-LDHs (Figure 7C). ${ }^{[79]}$ As stated previously, no simple relation between interlayer space and coercive field is reported for these materials. ${ }^{[20,47]}$ In overall, the magnetic results for the functionalized sample do not depict significant deviations from those found in conventional NiFe-LDHs. ${ }^{[15,24,62,83]}$

Mössbauer spectroscopy was carried out to give further insights on the magnetic nature of the system and the coordination environment.

\begin{tabular}{ccccccccc}
\hline Sample & $\begin{array}{c}\boldsymbol{\chi} \cdot \mathbf{T}_{\mathbf{r t}} \\
\left(\mathbf{e m u} \cdot \mathbf{K} \cdot \mathbf{m o l}^{-1}\right)\end{array}$ & $\begin{array}{c}\mathbf{C}_{\mathbf{S O}} \\
\left(\mathbf{e m u} \cdot \mathbf{K} \cdot \mathbf{m o l}^{-\mathbf{1}}\right)\end{array}$ & $\begin{array}{c}\mathbf{C} \\
\left(\mathbf{e m u} \cdot \mathbf{K} \cdot \mathbf{m o l}^{-1}\right)\end{array}$ & $\begin{array}{c}\boldsymbol{\theta} \\
(\mathbf{K})\end{array}$ & $\begin{array}{c}\mathbf{T}_{\mathrm{B}} \\
(\mathbf{K})\end{array}$ & $\begin{array}{c}\mathbf{T}_{\mathbf{M}} \\
(\mathbf{K})\end{array}$ & $\begin{array}{c}\mathbf{M}_{\mathbf{S}} \\
\left(\boldsymbol{\mu}_{\mathrm{B}}\right)\end{array}$ & $\begin{array}{c}\mathbf{H}_{\mathbf{c}} \\
(\mathbf{O e})\end{array}$ \\
\hline $\begin{array}{c}\mathbf{N i F e - C O} 3 \\
(\mathbf{H T})\end{array}$ & 2.29 & 2.10 & 2.56 & 29.1 & 15.1 & 16.8 & 0.76 & 3600 \\
\hline $\mathbf{N i F e - A Q}$ & 2.10 & 2.08 & 2.32 & -14.0 & 18.6 & 22.4 & 1.22 & 1300 \\
\hline $\mathbf{N i F e - D S}$ & 2.11 & 2.10 & 2.28 & -7.4 & 12.8 & 15.8 & 1.07 & 2500 \\
\hline $\begin{array}{c}\mathbf{N i F e}- \\
\text { APTES }\end{array}$ & 1.57 & 2.11 & 1.50 & 9.5 & 12.9 & 15.7 & 0.70 & 2700 \\
\hline
\end{tabular}

Table 4. Main magnetic data of the NiFe-LDH family.

$\chi \cdot \mathrm{T}_{\mathrm{rt}}$ value at room temperature; $\left(\mathrm{C}_{\mathrm{SO}}\right)$ expected spin-only value of the Curie constant; (C) experimental Curie constant; $(\theta)$ Weiss constant; $\left(T_{B}\right)$ blocking temperature; $\left(T_{M}\right)$ temperature for the onset of the spontaneous magnetization extracted from the $\chi_{\mathrm{M}}$ " plot; $\left(\mathrm{M}_{\mathrm{S}}\right)$ saturation magnetization; $\left(\mathrm{H}_{\mathrm{c}}\right)$ coercive field. 

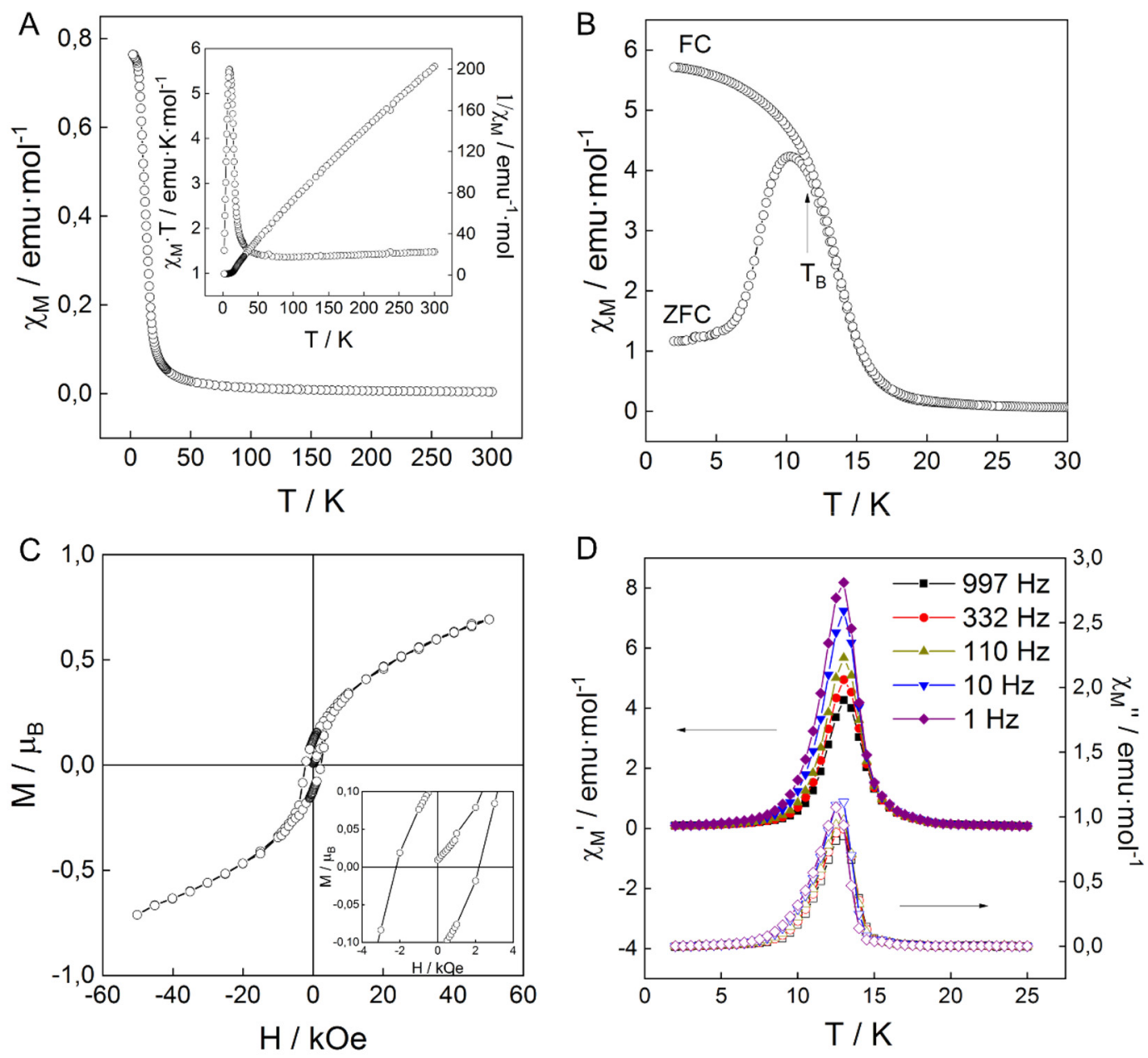

Figure 7. Magnetic properties of the NiFe-APTES sample. (A) $\chi_{M}$ vs. T plot at an applied field of 1000 Oe. The inset in represents the temperature dependence of the $\chi_{\mathrm{M}} \cdot \mathrm{T}$ product and the fitting of the magnetic data to a Curie-Weiss law. (B) FC/ZFC plot at an applied field of 100 Oe, the inset remarks the low field region highlighting the blocking temperature. (C) Hysteresis cycle at $2 \mathrm{~K}$. The inset highlights the low field region. (D) Thermal dependence of the $\chi^{\prime}{ }_{M}$ (in-phase) and $\chi{ }^{\prime}$ (out-of-phase) signals at 1, 10, 110, 332 and $997 \mathrm{~Hz}$.

As in the case of the $\mathrm{NiFe}-\mathrm{CO}_{3}$ compounds ${ }^{[15]}$ and in agreement with magnetization data, the Mössbauer spectra of the hybrid compounds NiFe-AQ, NiFe-DS and NiFe-APTES show a paramagnetic behavior at room temperature (Figure 8A), while at $4 \mathrm{~K}$ (Figure 8B), they reflect a slowing down of the relaxation of the Fe magnetic moments directions below the Larmor precession rate of the ${ }^{57} \mathrm{Fe}$ nuclei, indicating the presence of strong magnetic correlations in the solids. The absorption peaks of these compounds are however broader than those of the NiFe$\mathrm{CO}_{3}$ suggesting a wider diversity of $\mathrm{Fe}^{\mathrm{III}}$ environments, most probably due to different arrangements of the neighboring AQ, DS and APTES molecules.

As explained in detail in Ref. ${ }^{[15]}$, if the occupation of the cation sites by $\mathrm{Fe}^{\mathrm{III}}$ and $\mathrm{Ni}^{\mathrm{II}}$ is perfectly ordered, all the $\mathrm{Fe}$ cations coordinated by six $\mathrm{OH}^{-}$would also have the same second 
coordination sphere, i.e., the same number of $\mathrm{Ni}^{\mathrm{II}}$ and $\mathrm{Fe}^{\mathrm{III}}$ nearest neighbors. This would lead to only one sextet at $4 \mathrm{~K}$ and a single quadrupole doublet at $295 \mathrm{~K}$. This is not the case for the hybrid compounds whose spectra suggest a disordered cation distribution.

The room temperature spectra were analysed with a distribution of quadrupole splittings while those obtained at $4 \mathrm{~K}$ were fitted with a set of magnetic sextets. The number of sextets in each set was selected assuming that the effect on the magnetic hyperfine fields, $\mathrm{Bhf}_{\mathrm{hf}}$ of the number of $\mathrm{Ni}^{\mathrm{II}}$ nearest neighbours of the $\mathrm{Fe}^{\mathrm{III}}$ is dominant when compared to the effect of the different arrangements of the neighbour AQ, DS and APTES molecules. The differences in $B_{h f}$ are thus mainly related to the different number of $\mathrm{Ni}^{\mathrm{II}}$ nearest neighbors of the $\mathrm{Fe}^{\mathrm{III}}$, since the $\mathrm{Fe}-\mathrm{OH}-\mathrm{Fe}$ and $\mathrm{Fe}-\mathrm{OH}-\mathrm{Ni}$ superexchange interactions are not equal. ${ }^{[15]}$

Assuming a completely random distribution of $\mathrm{Fe}^{\mathrm{III}}$ and $\mathrm{Ni}^{\mathrm{II}}$ the probability of finding $m \mathrm{Ni}^{\mathrm{II}}$ cations in a shell of six nearest neighbour sites around $\mathrm{Fe}^{\mathrm{III}}$ is given by the binomial distribution function:

$$
P(m)=\frac{6 !}{m !(6-m) !} y^{m}(1-y)^{6-m}
$$

where $y$ stands for the ratio $\mathrm{Ni}^{\mathrm{II}}: \mathrm{Fe}^{\mathrm{III}}$. Considering the data from EDS, elemental analysis, XPS and TG-MS the $\mathrm{Ni}^{\mathrm{II}}: \mathrm{Fe}^{\mathrm{III}}$ ratio is 2.1 for NiFe-AQ and 2.0 for NiFe-DS and NiFe-APTES. The estimated $P(m)$ are summarized in Table S3. Considering that all the $\mathrm{Fe}^{\mathrm{III}}$ occupy the same crystallographic sites and have the same coordination -six $\mathrm{OH}^{-}-$they are expected to have similar recoil-free fractions. Therefore, the relative areas, $I$, estimated for the spectra at $4 \mathrm{~K}$ should be approximately equal to the fraction of $\mathrm{Fe}^{\mathrm{III}}$ cations contributing to the corresponding sextets. If the metal cations are randomly distributed, the estimated $I$ values should be close to the calculated probabilities $P(m)$. When $I \leq 2 \%$ the corresponding sextet is difficult to resolve from those with higher $I$ and similar $\mathrm{B}_{\mathrm{hf}}$ and isomer shift values. Therefore, the sextets with very small areas are discarded and their $I$ are added to the $I$ of those with the closest number of $\mathrm{Ni}^{\mathrm{II}}$ nearest neighbours. ${ }^{[15]}$ Five sextets were then used in the refinement of the spectra obtained at $4 \mathrm{~K}$ (Table S4). Their expected $I$ in the case of random occupation of the cation sites by $\mathrm{Fe}^{\mathrm{III}}$ and $\mathrm{Ni}^{\mathrm{II}}$ are summarized in Table S4. Since antiferromagnetic Fe-OH-Fe interactions are stronger than the $\mathrm{Fe}-\mathrm{OH}-\mathrm{Ni}$ ones, $\mathrm{B}_{\mathrm{hf}}$ are expected to increase with increasing number of $\mathrm{Fe}^{\mathrm{III}}$ nearest neighbours, i.e. with decreasing $m$.

The estimated isomer shifts at 4 and $295 \mathrm{~K}$ (Table S5), consistent with high-spin ( $\mathrm{S}=5 / 2$ ) $\mathrm{Fe}^{\mathrm{III}}$, are the same, within experimental error, for all the compounds.

The calculated average $\mathrm{B}_{\text {hf }}$ values at $4 \mathrm{~K}$ are also consistent with high-spin $\mathrm{Fe}^{\mathrm{III}}$ although lower than those of the $\mathrm{NiFe}-\mathrm{CO}_{3}$. This may be related to the significantly different interlamellar spacing between $\mathrm{NiFe}-\mathrm{CO}_{3}, \sim 7.8 \AA,^{[15]}$ and the hybrid ones, in the range $20.8-27.1 \AA$. 
The comparison of the theoretical $I$ values in Table $\mathbf{S} 4$ with the set of experimental $I$ calculated from the spectra (Table S5) suggests that the $\mathrm{Ni}^{\mathrm{II}}$ and $\mathrm{Fe}^{\mathrm{III}}$ cation distribution is not completely random. In the case of sextets corresponding to a higher number of $\mathrm{Fe}^{\mathrm{III}}$ nearest neighbours, the observed $I$ values are higher than in the case of a completely random distribution (compare Tables S4 and S5). Obviously the reverse is observed for sextets corresponding to a lower number of $\mathrm{Fe}^{\mathrm{III}}$ whose $I$ values are smaller than those calculated assuming a completely random distribution. This deviation suggests that there is a tendency for $\mathrm{Fe}^{\mathrm{III}}$ clustering as already observed in $\mathrm{NiFe}-$ $\mathrm{CO}_{3}$.

According to Mössbauer data the functionalization in the NiFe-APTES compound did not affect the electronic state of $\mathrm{Fe}^{\mathrm{III}}$ and had virtually no effect on the overall magnetic behavior when compared to conventional NiFe-LDHs.
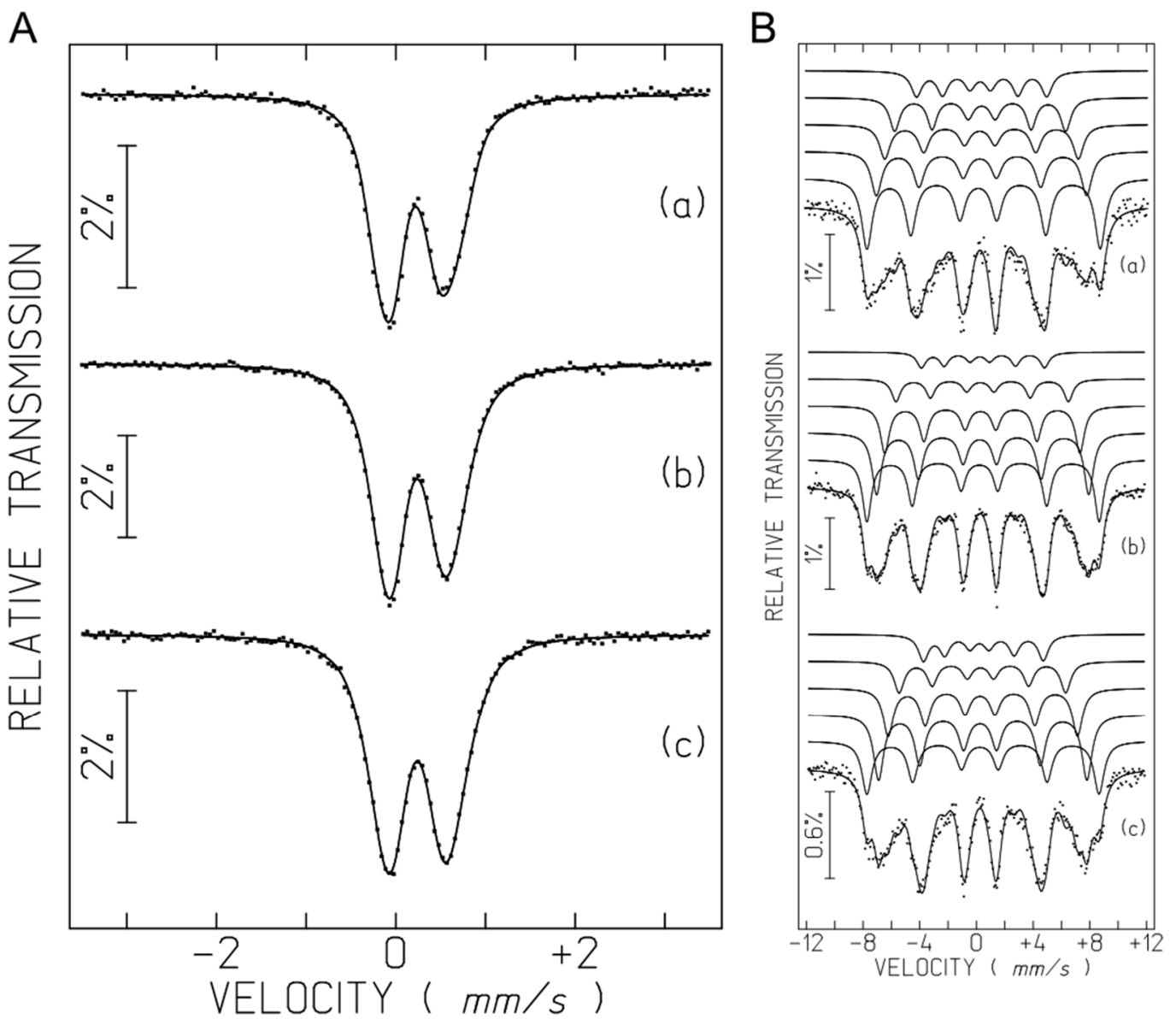

Figure 8. (A) Mössbauer spectra of the NiFe-LDHs (a) - AQ (b) -DS (c) -APTES taken at room temperature. The lines over the experimental points are distributions of quadrupole splittings. The estimated parameters are collected in Table S5. (B) Mössbauer spectra of the NiFe-LDHs (a) -AQ (b) -DS (c) -APTES taken at $4 \mathrm{~K}$. The lines over the experimental points are the sum of sextets 
corresponding to $\mathrm{Fe}^{\mathrm{III}}$ atoms with different number of $\mathrm{Ni}^{\mathrm{II}}$ nearest neighbours. The estimated parameters for these sextets, shown slightly shifted for clarity, are collected in Table S5.

Beyond magnetism, we are also interested in exploring how the covalent functionalization can enhance their anion exchange capability. Since this property has a potential use towards the removal of contaminant anions. ${ }^{[84,85]}$ Mainly, the overall physicochemistry of the anion exchange reactions are controlled by charge density. ${ }^{[86,87]}$ This value depends on the intralayer parameter (a) given by the cationic radii and the $\mathrm{M}^{\mathrm{III}}$ composition $(x)$. In general terms, the highest the $x$, the highest the anionic amount absorbed. Whatever the case, the maximum amount of anion in the interlayer space depends on the $x$ value (electroneutrality). Keeping in mind the nature of the employed functionalization in which more than $65 \%$ of the amine groups are positively charged as $-\mathrm{NH}_{3}{ }^{+}$(stated by the XPS results), we performed a set of anion exchange reactions over the $\mathrm{NiFe}-\mathrm{AQ}$, $\mathrm{NiFe}-\mathrm{DS}$ and $\mathrm{NiFe}-\mathrm{APTES}$ with $\mathrm{HCrO}_{4}{ }^{-}$anions in order to complex $\mathrm{Cr}(\mathrm{VI}) .{ }^{[88]}$ Only $\mathrm{NiFe}-$ APTES exhibited a successful intercalation of $\mathrm{HCrO}_{4}^{-}$. Figure $\mathbf{S 1 2}$ depicts the EDS measurement confirming the presence of $\mathrm{Cr}(\mathrm{VI})$ as well as the absence of traces of $\mathrm{Cl}$ or $\mathrm{Br}$, pointing out the complete incorporation of desired anion. XRPD measurement recorded over the NiFe-APTES sample confirms that the functionalized LDH phase is conserved after the insertion of the contaminant anion, while the diffractograms of $\mathrm{NiFe}-\mathrm{AQ}$ and $\mathrm{NiFe}-\mathrm{DS}$ remain unaltered after the unsuccessful anion exchange (Figure S13). Furthermore, the Cr:Fe ratio was found to be 2.3 for the functionalized sample. This result could be understood as a consequence of the presence of $-\mathrm{NH}_{3}{ }^{+}$groups, which need a counter anion to compensate the positive charge, enhancing the adsorption property of the $\mathrm{LDH}$. In addition, the $\mathrm{Cr}:(\mathrm{Fe}+\mathrm{Si})$ ratio close to one confirms this hypothesis. In this line, these results show an increment in the retention of $\mathrm{Cr}(\mathrm{VI})$ moieties in $c a .230 \%$ with respect to a typical $\mathrm{LDH}$, in which the maximum $\mathrm{Cr}: \mathrm{Fe}$ ratio is expected to be 1 (depending on the $x$ value to keep the electroneutrality). These results pave the way to the synthesis of LDHs with a higher degree of retention regarding to anionic contaminants such as $\mathrm{Cr}(\mathrm{VI})$ or $\mathrm{As}(\mathrm{V})$, among others. ${ }^{[88]}$

Finally, we investigated the reversibility of the covalent grafting by $\mathrm{pH}$-induced retrofunctionalization. In this sense, it is important to point out that the silane functionalization is not compatible with a strong alkali media $(\mathrm{pH}: 13-14)$, since at basic $\mathrm{pH}$ the silica's solubility strongly increases. ${ }^{[89]}$ In order to further analyse this behaviour, a NiFe-APTES suspension adjusted at $\mathrm{pH}=13$ with $\mathrm{NaOH}$ was prepared and left overnight under permanent magnetic stirring. Figure S14 depicts the XRPD pattern and FTIR spectrum recorded over the solid sample after the basic treatment. The diffractogram shows the typical peaks attributed to a LDH phase of both inter and intralayer distances. In the case of the indexed peaks (003) and (006), an interlayer distance of $7.8 \AA$ can be calculated, typically obtained for $\mathrm{CO}_{3}{ }^{2-}$ and $\mathrm{OH}^{-}$as interlayer anions, thus indicating the loss of the APTES molecules. At the same time, no changes were observed in 
terms of intralayer distance (indexed as 110) confirming the identity of the LDH sample. Regarding the infrared spectrum, no signal of the APTES bands are found. Instead, the typical carbonate peaks can be observed, ${ }^{[62]}$ in good agreement with the XRPD diffractogram. EDS probe confirms the absence of $\mathrm{Si}$, pointing out the complete loss of the functionalization, while keeping intact the $\mathrm{Ni}: \mathrm{Fe}$ ratio.

Taking this into account, it is expected that the reversibility of silane bonding should not affect the excellent electrocatalytic behaviour of NiFe-LDHs. ${ }^{[24,25,27]}$ In this context, we tested the oxygen evolution reaction (OER) electrocatalytic performance of NiFe-AQ and NiFe-APTES in a basic medium (1 M KOH) in a standard three-electrode cell (Figure 9 and Table 5). Both samples were measured by cyclic voltammetry (Figure S15 and S16) and linear sweep voltammetry (LSV), showing an excellent electrochemical performance. The polarization curves, depicted in Figure 9A, show low onset potentials of $344 \mathrm{mV}$ for $\mathrm{NiFe}-\mathrm{AQ}$ and $354 \mathrm{mV}$ for $\mathrm{NiFe}-$ APTES of overpotential (1.570 V and 1.580 vs the reversible hydrogen electrode (RHE)). An overpotential of $275 \mathrm{mV}$ is required at $j=10 \mathrm{~mA} \mathrm{~cm}^{-2}$ for NiFe-AQ, a value very close to that of $\mathrm{NiFe}-\mathrm{APTES}(270 \mathrm{mV})$. However, if the current density is increased to $j=30 \mathrm{~mA} \mathrm{~cm}{ }^{-2}, \mathrm{NiFe}-$ AQ requires an overpotential of $317 \mathrm{mV}$, a bit lower than that for NiFe-APTES (326 mV). In addition, a maximum current density of $715 \mathrm{~mA} \mathrm{~cm}{ }^{-2}$ is found at $800 \mathrm{mV}$ of overpotential. Moreover, compared to typical carbonate-intercalated NiFe-LDH (synthesized by hydrothermal approach) shows an onset potential of $369 \mathrm{mV}$ overpotential, around $15 \mathrm{mV}$ higher than the other samples. In addition, the overpotential required for a current density of 10 and $30 \mathrm{~mA} \cdot \mathrm{cm}^{-2}$ are 277 and $326 \mathrm{mV}$, respectively (Figure S17). Therefore, we can say that the carbonate-intercalated NiFe-LDH offers a comparable behaviour, with slightly worse onset potential and similar values of overpotential at different current densities. The excellent catalytic activity of both $\mathrm{NiFe}-\mathrm{AQ}$ and NiFe-APTES samples is also reflected in the Tafel slopes (Figure 9B), showing values of 57 and $58 \mathrm{mV}$ per decade, respectively. Finally, the stability and durability of the materials were tested at constant current densities $j$ of 10 and $15 \mathrm{~mA} \cdot \mathrm{cm}^{-2}$ and with a constant overpotential, $\eta$, of 300 and $400 \mathrm{mV}$ for $10000 \mathrm{~s}$. Figure 9C displays the stability of both samples, highlighting a very good stability. According to these data, it is clearly demonstrated that the retrofunctionalization of the silane molecules at basic $\mathrm{pHs}$ give rise to very similar results compared to the non-covalent $\mathrm{NiFe}-\mathrm{AQ}$ materials, and pave the way for the covalent-assisted processing of $\mathrm{NiFe}-\mathrm{LDHs}$ into more complex architectures and its ulterior application in energy storage and conversion. 

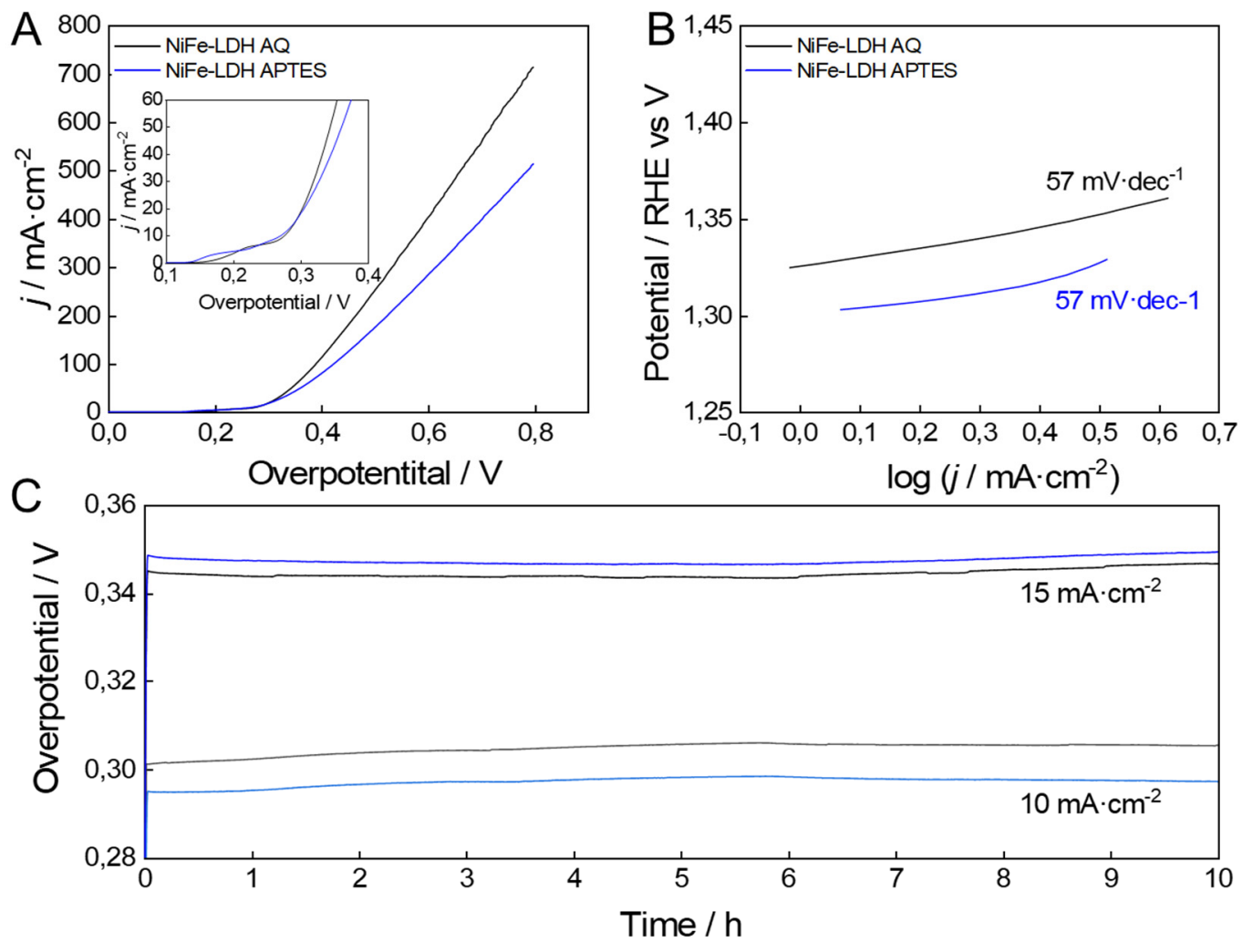

Figure 9. (A) Polarization curves of NiFe-AQ (black line) and NiFe-APTES (blue line) in $1 \mathrm{M} \mathrm{KOH}$. Inset show the polarization curves at low current densities. (B) Tafel plots and values of Tafel slopes. (C) The potentiostatic and galvanostatic stability testing under a certain potential and current densities.

\begin{tabular}{cccc}
\hline Sample & $\begin{array}{c}\text { Overpotential at } \\
\mathbf{1 0} \mathbf{~ m A} \cdot \mathbf{c m}^{-\mathbf{2}} / \mathbf{~} \mathbf{V V}\end{array}$ & $\begin{array}{c}\text { Overpotential at } \\
\mathbf{3 0} \mathbf{~} \mathbf{A} \cdot \mathbf{c m}^{-\mathbf{2}} \mathbf{\mathbf { m V }}\end{array}$ & $\begin{array}{c}\text { Tafel Slope / } \\
\mathbf{m V} \cdot \mathbf{d e c}^{-\mathbf{1}}\end{array}$ \\
\hline $\mathrm{NiFe}-\mathrm{AQ}$ & 275 & 317 & 57 \\
$\mathrm{NiFe}-\mathrm{APTES}$ & 270 & 326 & 58 \\
\hline
\end{tabular}

Table 5. Electrochemical data of the NiFe-AQ and NiFe-APTES samples.

\section{Conclusions}

In summary, we have reported here the covalent functionalization of a $\mathrm{NiFe}-\mathrm{LDH}$ with APTES molecules thanks to a topochemical approach supported with anion exchange reactions. The as-synthesized NiFe-APTES sample exhibits a successful LDH phase with the presence of APTES grafted within its structure. Thanks to solid state ${ }^{29} \mathrm{Si}$ NMR we gained further insights into the grafting modes of the silane molecules to the LDH layers, with a clear predominance of 
the monodentate and bidentate modes over the tridentate one. Furthermore, a TG-MS analysis, barely explored in the LDH field, was carried out to identify the different moieties in the LDH structure. This confirmed the loss of the functionalization when the LDH structure collapses. A complete magnetic analysis combined with Mössbauer spectroscopy showed slight deviations regarding the overall magnetic behavior, as compared to conventional $\mathrm{NiFe-LDHs.} \mathrm{This} \mathrm{indicates}$ that the electronic properties of the LDH layers are not affected by their covalent functionalization. Furthermore, the retention capacity towards anionic contaminants $\left(\mathrm{HCrO}_{4}^{-}\right.$; $\mathrm{Cr}^{\mathrm{VI}}$ ) was increased in more than $200 \%$ thanks to the grafted amino moieties. Finally, the reversibility of the silane-functionalization at basic $\mathrm{pH}$ was demonstrated, and the quality of the resulting $\mathrm{NiFe}-\mathrm{LDH}$ proved by studying the electrochemical performance in the oxygen evolution reaction in basic media. This capability of reversibly grafting molecules in a covalent way without a significant alteration of the most fundamental, structural and electronic LDH properties, while adding new improved functionalities, opens the door to a new degree of chemical tunability in LDHs. This work grounds the field towards the assembly of more complex architectures and hybrid materials based on LDHs.

\section{Experimental}

\section{Chemicals}

$\mathrm{NiCl}_{2} \cdot 6 \mathrm{H}_{2} \mathrm{O}, \mathrm{FeCl}_{2} \cdot 4 \mathrm{H}_{2} \mathrm{O}$, hexamethylenetetramine $\left(\mathrm{C}_{6} \mathrm{H}_{12} \mathrm{~N}_{4}, \mathrm{HMT}\right)$, anthraquinone-2sulfonic acid sodium salt monohydrate $\left(\mathrm{C}_{14} \mathrm{H}_{7} \mathrm{NaO}_{5} \mathrm{~S} \cdot \mathrm{H}_{2} \mathrm{O}\right)$, sodium dodecyl sulphate $\left(\mathrm{C}_{12} \mathrm{H}_{25} \mathrm{NaO}_{4} \mathrm{~S}\right)$, hexadecyltrimethylammonium bromide $\left(\mathrm{C}_{19} \mathrm{H}_{42} \mathrm{BrN}, \quad \mathrm{CTAB}\right)$, (3aminopropyl)triethoxysilane $\left(\mathrm{C}_{9} \mathrm{H}_{23} \mathrm{NO}_{3} \mathrm{Si}\right.$, APTES) and potassium dichromate $\left(\mathrm{K}_{2} \mathrm{Cr}_{2} \mathrm{O}_{7}\right)$ were purchased from Sigma-Aldrich. Ethanol absolute $\left(\mathrm{C}_{2} \mathrm{H}_{6} \mathrm{O}\right.$, EtOH), hydrochloric acid $(\mathrm{HCl})$ dichloromethane $\left(\mathrm{CHCl}_{2}, \mathrm{DCM}\right)$ and toluene $\left(\mathrm{C}_{7} \mathrm{H}_{8}\right)$ were purchased from Scharlab. Ultrapure water was obtained from a Millipore Milli-Q equipment.

\section{Synthesis of NiFe-LDH family}

To obtain the final $\mathrm{NiFe}-\mathrm{APTES}$ product, a series of anionic exchange reactions on the pristine $\mathrm{NiFe}-\mathrm{AQ}$ LDH material were carried out.

NiFe-anthraquinone (NiFe-AQ) LDH was synthesized according with the topochemical oxidation method reported elsewhere. ${ }^{[23,41]}$ In a typical synthesis, $5 \mathrm{mM}$ of $\mathrm{NiCl}_{2} \cdot 6 \mathrm{H}_{2} \mathrm{O}$ and 2.5 $\mathrm{mM}$ of $\mathrm{FeCl}_{2} \cdot 4 \mathrm{H}_{2} \mathrm{O}$ were mixed with $5 \mathrm{mM}$ of anthraquinone-2-sulfonic acid sodium salt monohydrate and $60 \mathrm{mM}$ of HMT and dissolved in $500 \mathrm{~mL}$ of Milli-Q water. The solution was then refluxed under Ar atmosphere and magnetic stirring for $6 \mathrm{~h}$. The resulting mixture was filtered and washed with Milli-Q water and ethanol, dried at room temperature and collected as the final yellowish product. 
NiFe-dodecyl sulphate (NiFe-DS) LDH was prepared from the NiFe-AQ LDH. In a typical reaction, $200 \mathrm{mg}$ of $\mathrm{NiFe}-\mathrm{AQ} \mathrm{LDH}$ were dispersed in $200 \mathrm{~mL}$ of a $\mathrm{H}_{2} \mathrm{O} / \mathrm{EtOH}(1: 1$, v/v) binary solution with $0.2 \mathrm{M}$ of SDS and $2 \mathrm{mM} \mathrm{HCl}$. The mixture was exposed under magnetic stirring and $\mathrm{Ar}$ atmosphere for $24 \mathrm{~h}$. The resulting solution was filtered, washed, dried at room temperature and finally collected as the previous sample.

NiFe-(3-Aminopropyl)triethoxysilane (NiFe-APTES) LDH was synthesized by modifying the method described by Kim and coworkers. ${ }^{[33]}$ Firstly, CTAB and the NiFe-DS LDH were dried overnight under vacuum at $80^{\circ} \mathrm{C}$ in different vials to remove moisture. Then, $30 \mathrm{~mL}$ of DCM was added to $1.75 \mathrm{~g}$ of CTAB $(0.16 \mathrm{M})$ under Ar atmosphere, followed with the heating at $50^{\circ} \mathrm{C}$ until the complete solution of CTAB. Separately, $5 \mathrm{~mL}$ of APTES were added to the NiFe-DS LDH and mixed with the CTAB+DCM solution. The final mixture was kept at $50{ }^{\circ} \mathrm{C}$ under inert atmosphere and magnetic stirring for $48 \mathrm{~h}$. Finally, the product was filtered and washed thoroughly with DCM, dried at room temperature, grinded and collected.

\section{$\operatorname{Cr}(\mathrm{VI})$ anion exchange reactions}

A suspension of NiFe-APTES in Milli-Q water $\left(1 \mathrm{~g} \cdot \mathrm{L}^{-1}\right)$ was prepared and dispersed by bath sonication for $30 \mathrm{~min}$. Subsequently, $\mathrm{K}_{2} \mathrm{Cr}_{2} \mathrm{O}_{7}(36.7 \mathrm{mg}$ in $5 \mathrm{~mL})$ was added reaching a final concentration of $50 \mathrm{mM}$, and the resulting suspension was stirred under ambient conditions for 24 hours. Finally, the $\mathrm{NiFe}-\mathrm{APTES}+\mathrm{HCrO}_{4}^{-}$sample was isolated by ultracentrifugation a 13400 r.p.m. for $5 \mathrm{~min}$ and thoroughly washed with Milli-Q water for 3 times.

\section{Electrochemical characterization}

The electrochemical experiments were performed using an Autolab potenciostat (Autolab $128 \mathrm{~N}$ potentiostat/galvanostat) connected to a personal computer that uses NOVA 2.1 electrochemical software. The powdered materials were mixed with acetylene black and PVDF in a mass ratio of 80:10:10 in ethanol and deposited on a nickel foam electrode. The as-prepared nickel foam electrodes were dried overnight at $80{ }^{\circ} \mathrm{C}$ and pressed at $1000 \mathrm{kPa}$. Each working electrode contained about $0.25 \mathrm{mg} \cdot \mathrm{cm}^{-2}$ of electroactive material and had a geometric surface area of about $0.2 \mathrm{~cm}^{2}$. A typical three-electrode experimental cell equipped with a stainless steel plate having $4 \mathrm{~cm}^{2}$ of surface area as the counter electrode, and a Metrohm $\mathrm{Ag} / \mathrm{AgCl}(3 \mathrm{M} \mathrm{KCl})$ as the reference electrode was used for the electrochemical characterization of the working electrodes. All measurements were carried out after $10 \mathrm{~min}$ of magnetic stirring under nitrogen bubbling. The electrochemical properties were studied measuring the cyclic voltammetry (CV) at different scan rates and linear sweep voltammetry (LSV) using $1 \mathrm{M} \mathrm{KOH}$ aqueous solutions. In addition, chronoamperometric studies were performed at a constant overpotential and chronopotentiometric studies at constant current densities. All potentials reported in this 
manuscript were converted to the overpotential $(\mathrm{OP})$ scale, using: $\mathrm{E}_{(\mathrm{OP})}=\mathrm{E}_{(\mathrm{NHE})}-0.4 \mathrm{~V}=$ $\mathrm{E}_{(\mathrm{Ag} / \mathrm{AgCl})}^{\mathrm{o}}+0.197 \mathrm{~V}-0.4 \mathrm{~V}$

\section{Physical characterization}

X-ray powder diffraction (XRPD) patterns were obtained with a Philips X'Pert diffractometer using the copper radiation $(\mathrm{Cu}-\mathrm{K} \alpha=1.54178 \AA$ ). Diffractograms were recorded in the capillary with no preferred orientation. ATR Infrared spectra were recorded with an Agilent Cary 630 FTIR spectrometer in the 4000-650 $\mathrm{cm}^{-1}$ range with no need of $\mathrm{KBr}$ pellets. Nitrogen adsorption/desorption isotherms at $77 \mathrm{~K}$ were collected in an AUTOSORB-6 apparatus. Samples ( $>100 \mathrm{mg}$ for each sample) were previously degassed at $423 \mathrm{~K}$ for $12 \mathrm{~h}$ under vacuum with an AUTOSORB DEGASSER. Dynamic light scattering (DLS) measurements were recorded at 25 ${ }^{\circ} \mathrm{C}$ with a Zetasizer Nano ZS instrument from Malvern Instrument Ltd. Field emission scanning electron microscopy (FESEM) studies were carried out on a Hitachi S-4800 microscope at an accelerating voltage of $20 \mathrm{kV}$ and 30 seconds of $\mathrm{Au} / \mathrm{Pd}$ metallization of the samples. Highresolution transmission electron microscopy (HRTEM) studies were carried out on Tecnai G2 F20 microscope operating at $200 \mathrm{kV}$. Samples were prepared by dipping a sonicated suspension of the sample in ethanol on a carbon-coated copper grid. Metallic atomic composition of bulk samples was determined by means of electron probe microanalysis (EPMA) performed in a Philips SEM-XL30 equipped with an EDS microprobe. Elemental analysis (EA) for carbon, nitrogen and hydrogen contents were determined by microanalytical procedures by using a LECO CHNS. Thermogravimetric analysis (TGA) coupled with a mass spectrometer (MS) was performed on a Netzsch STA 409 CD instrument equipped with a Skimmer QMS 422 mass spectrometer (MS/EI) with the following programmed time-dependent temperature profile: $25-$ $800{ }^{\circ} \mathrm{C}$ with $10{ }^{\circ} \mathrm{C} \cdot \mathrm{min}^{-1}$ gradient and cooling to room temperature under $\mathrm{He}$ atmosphere. The initial sample weights were about $5 \mathrm{mg}$, and the whole experiment was performed under helium with a gas flow of $80 \mathrm{~mL} \cdot \mathrm{min}^{-1}$. X-ray photoelectron spectroscopy (XPS) measurements were performed in an ultrahigh vacuum system ESCALAB210 (base pressure 1.0 $\times 10-10$ mbar) from Thermo VG Scientific. Photoelectrons were excited by using the $\mathrm{Mg}-\mathrm{K} \alpha$ line (1253.6 eV). All spectra were referred to the Fermi level. ${ }^{29} \mathrm{Si}$ NMR spectra was collected using a Bruker DRX 300 console, with a magnet operating at $7 \mathrm{~T}$. The corresponding ${ }^{29} \mathrm{Si}$ frequency was $59.6 \mathrm{MHz}$. A custom-built probe head equipped with a solenoid coil and tunable capacitors was used to maximize sensitivity and filling. About $100 \mu \mathrm{l}$ of chloromethyl(dimethyl)silane was used as reference. This reference has a chemical shift of -200 to $-300 \mathrm{ppm}$ from tetramethylsilane (TMS). About $100 \mathrm{mg}$ of LDH samples were put in a Teflon sample holder, sealed with Teflon tape to avoid moisture uptake. Each spectrum contains an average of 30000 FIDs. After the summation a Savitzky-Golay filter of 20 points to the $2^{\text {nd }}$ polynomial order was applied to filter out statistical noise. The pulse length was $6 \mu \mathrm{s}$, power was $25 \mathrm{~W}$. The repetition rate was varied between $0.1 \mathrm{~s}$ 
to $10 \mathrm{~s}$. Magnetic data were collected with a Quantum Design superconducting quantum interference device (SQUID) MPMS-XL-5. The susceptibility data were corrected from the diamagnetic contributions of the atomic constituents of the samples as deduced from Pascal's constant tables and the sample holder. The DC data were obtained under an external applied field of 100 or $1000 \mathrm{Oe}$ in the $2-300 \mathrm{~K}$ temperature range. Magnetization studies were performed between -5 and $+5 \mathrm{~T}$ at a constant temperature of $2 \mathrm{~K}$. The AC data were collected under an applied field of 3.95 Oe at 997, 333, 110, 10 and $1 \mathrm{~Hz}$. Mössbauer spectra were collected in transmission mode using a conventional constant-acceleration spectrometer and a $25 \mathrm{mCi}{ }^{57} \mathrm{Co}$ source in a Rh matrix. The velocity scale was calibrated using $\alpha$-Fe foil. The absorbers were obtained by packing the powdered samples into perspex holders. Isomer shifts are given relative to metallic $\alpha$-Fe at room temperature. The spectra at $4.1 \mathrm{~K}$ were collected using a bath cryostat with the sample immersed in liquid He. The spectra were fitted to Lorentzian lines using a nonlinear least-squares method. ${ }^{[90]}$ Distributions of quadrupole splitting were fitted according to the histogram method. ${ }^{[1]}$

\section{Acknowledgements}

This work was partially supported by the European Research Council (ERC Advanced Grant Mol-2D 788222 to E.C. and ERC Starting Grant 2D-PnictoChem 804110 to G.A.). G.A. thanks support by the Deutsche Forschungsgemeinschaft (DFG; FLAG-ERA AB694/2-1), and the Generalitat Valenciana (SEJI/2018/034 grant and CIDEGENT/2018/001). J.A.C. thanks the ERC Starting Grant 2D-PnictoChem for the postdoctoral contract. This work was supported by the Spanish MINECO (Unit of Excellence "Maria de Maeztu" MDM-2015-0538 and Project MAT2017-89993-R co-financed by FEDER), and Generalitat Valenciana (Prometeo/2017/066). C2TN/IST authors gratefully acknowledge the FCT support through the UID/Multi/04349/2013 project. B. G. M. and F. S. were supported by the National Research, Development and Innovation Office of Hungary (NKFIH) Grant Nrs. K119442 and 2017-1.2.1-NKP-2017-00001. We also want to acknowledge Vicent Lloret for his help with the TG-MS measurements, as well as Frank Hauke and Andreas Hirsch for the use of the equipment into their facilities. 


\section{References}

[1] A. K. Geim, K. S. Novoselov, Nat. Mater. 2007, 6, 183-191.

[2] S. Z. Butler, S. M. Hollen, L. Cao, Y. Cui, J. A. Gupta, H. R. Gutiérrez, T. F. Heinz, S. S. Hong, J. Huang, A. F. Ismach, et al., ACS Nano 2013, 7, 2898-2926.

[3] A. K. Geim, I. V. Grigorieva, Nature 2013, 499, 419-425.

[4] V. Nicolosi, M. Chhowalla, M. G. Kanatzidis, M. S. Strano, J. N. Coleman, Science 2013, $340,1226419$.

[5] D. Hanlon, C. Backes, E. Doherty, C. S. Cucinotta, N. C. Berner, C. Boland, K. Lee, A. Harvey, P. Lynch, Z. Gholamvand, et al., Nat. Commun. 2015, 6, 8563.

[6] G. Abellán, S. Wild, V. Lloret, N. Scheuschner, R. Gillen, U. Mundloch, J. Maultzsch, M. Varela, F. Hauke, A. Hirsch, J. Am. Chem. Soc. 2017, 139, 10432-10440.

[7] C. Gibaja, D. Rodriguez-San-Miguel, P. Ares, J. Gómez-Herrero, M. Varela, R. Gillen, J. Maultzsch, F. Hauke, A. Hirsch, G. Abellán, et al., Angew. Chem. Int. Ed. 2016, 55, 1434514349.

[8] P. Ares, J. J. Palacios, G. Abellán, J. Gómez-Herrero, F. Zamora, Adv. Mater. 2017, 1703771 .

[9] Q. H. Wang, K. Kalantar-Zadeh, A. Kis, J. N. Coleman, M. S. Strano, Nat. Nanotechnol. 2012, 7, 699-712.

[10] A. K. Geim, I. V. Grigorieva, Nature 2013, 499, 419-425.

[11] J. López-Cabrelles, S. Mañas-Valero, I. J. Vitórica-Yrezábal, P. J. Bereciartua, J. A. Rodríguez-Velamazán, J. C. Waerenborgh, B. J. C. Vieira, D. Davidovikj, P. G. Steeneken, H. S. J. van der Zant, et al., Nat. Chem. 2018, 10, 1001-1007.

[12] S. M. Auerbach, K. A. Carrado, P. K. Dutta, Handbook of Layered Materials, CRC Press, 2004.

[13] Z. Liu, R. Ma, M. Osada, N. Iyi, Y. Ebina, K. Takada, T. Sasaki, J. Am. Chem. Soc. 2006, $128,4872-4880$.

[14] X. Duan, D. G. Evans, Layered Double Hydroxides, Springer, 2006.

[15] G. Abellán, E. Coronado, C. Martí-Gastaldo, J. Waerenborgh, A. Ribera, Inorg. Chem. 2013, 52, 10147-10157.

[16] Q. Wang, D. O'Hare, Chem. Rev. 2012, 112, 4124-4155. 
[17] J. Liang, R. Ma, N. Iyi, Y. Ebina, K. Takada, T. Sasaki, Chem. Mater. 2010, 22, 371378.

[18] J. A. Carrasco, A. Harvey, D. Hanlon, V. Lloret, D. McAteer, R. Sanchis-Gual, A. Hirsch, F. Hauke, G. Abellán, J. N. Coleman, et al., Chem. Commun. 2019, 55, 3315-3318.

[19] E. Coronado, C. Martí-Gastaldo, E. Navarro-Moratalla, A. Ribera, S. J. Blundell, P. J. Baker, Nat. Chem. 2010, 2, 1031-1036.

[20] J. A. Carrasco, S. Cardona-Serra, J. M. Clemente-Juan, A. Gaita-Ariño, G. Abellán, E. Coronado, Inorg. Chem. 2018, 57, 2013-2022.

[21] X. Guo, F. Zhang, D. G. Evans, X. Duan, Chem. Commun. 2010, 46, 5197-5210.

[22] M. Gong, Y. Li, H. Wang, Y. Liang, J. Z. Wu, J. Zhou, J. Wang, T. Regier, F. Wei, H. Dai, J. Am. Chem. Soc. 2013, 135, 8452-8455.

[23] W. Ma, R. Ma, C. Wang, J. Liang, X. Liu, K. Zhou, T. Sasaki, ACS Nano 2015, 9, $1977-$ 1984.

[24] J. A. Carrasco, J. Romero, M. Varela, F. Hauke, G. Abellán, A. Hirsch, E. Coronado, Inorg Chem Front 2016, 3, 478-487.

[25] F. Dionigi, P. Strasser, Adv. Energy Mater. 2016, 6, 1600621.

[26] Y. Wang, D. Yan, S. El Hankari, Y. Zou, S. Wang, Adv. Sci. 2018, 5, 1800064.

[27] J. A. Carrasco, R. Sanchis-Gual, A. Seijas-Da Silva, G. Abellán, E. Coronado, Chem. Mater. 2019, 31, 6798-6807.

[28] H. Yang, F. Li, C. Shan, D. Han, Q. Zhang, L. Niu, A. Ivaska, J. Mater. Chem. 2009, 19, $4632-4638$.

[29] V. Georgakilas, M. Otyepka, A. B. Bourlinos, V. Chandra, N. Kim, K. C. Kemp, P. Hobza, R. Zboril, K. S. Kim, Chem. Rev. 2012, 112, 6156-6214.

[30] S. Wild, M. Fickert, A. Mitrovic, V. Lloret, C. Neiss, J. A. Vidal-Moya, M. Á. RiveroCrespo, A. Leyva-Pérez, K. Werbach, H. Peterlik, et al., Angew. Chem. Int. Ed. 2019, 58, 57635768.

[31] P. C. Ma, J.-K. Kim, B. Z. Tang, Compos. Sci. Technol. 2007, 67, 2965-2972.

[32] T. D. Sargeant, M. S. Rao, C.-Y. Koh, S. I. Stupp, Biomaterials 2008, 29, 1085-1098.

[33] A.-Y. Park, H. Kwon, A. J. Woo, S.-J. Kim, Adv. Mater. 2005, 17, 106-109.

[34] W. Guo, Y. Zhao, F. Zhou, X. Yan, B. Fan, R. Li, Appl. Catal. Gen. 2016, 522, 101-108.

[35] Q. Tao, J. Yuan, R. L. Frost, H. He, P. Yuan, J. Zhu, Appl. Clay Sci. 2009, 45, 262-269. 
[36] L. Su, Q. Tao, H. He, J. Zhu, P. Yuan, Mater. Chem. Phys. 2012, 136, 292-295.

[37] H. He, Q. Tao, J. Zhu, P. Yuan, W. Shen, S. Yang, Appl. Clay Sci. 2013, 71, 15-20.

[38] L. Su, Q. Tao, H. He, J. Zhu, P. Yuan, R. Zhu, J. Colloid Interface Sci. 2013, 391, 1620.

[39] W. Ma, R. Ma, C. Wang, J. Liang, X. Liu, K. Zhou, T. Sasaki, ACS Nano 2015, 9, $1977-$ 1984.

[40] K. Yan, T. Lafleur, J. Chai, C. Jarvis, Electrochem. Commun. 2016, 62, 24-28.

[41] J.-H. Lee, D. O'Hare, D.-Y. Jung, Bull. Korean Chem. Soc. 2012, 33, 725-727.

[42] M. Meyn, K. Beneke, G. Lagaly, Inorg. Chem. 1993, 32, 1209-1215.

[43] A. Mendiboure, R. Schoellhorn, Rev Chim Min. 1987, 18, 819-827.

[44] F. Song, X. Hu, Nat. Commun. 2014, 5, 1-9.

[45] J. Zhu, P. Yuan, H. He, R. Frost, Q. Tao, W. Shen, T. Bostrom, J. Colloid Interface Sci. 2008, 319, 498-504.

[46] M. Ogawa, M. Hiramine, Cryst. Growth Des. 2014, 14, 1516-1519.

[47] C. Zhang, T. Tsuboi, H. Namba, Y. Einaga, T. Yamamoto, Dalton Trans 2016, 45, 13324-13331.

[48] M. Sahoo, S. Singha, K. M. Parida, New J. Chem. 2011, 35, 2503.

[49] G. R. Williams, D. O'Hare, J. Mater. Chem. 2006, 16, 3065.

[50] G. Lagaly, Clay Miner. 1981, 16, 1-21.

[51] H. He, R. L. Frost, F. Deng, J. Zhu, X. Wen, P. Yuan, Clays Clay Miner. 2004, 52, 350356.

[52] J. Pisson, C. Taviot-Guého, Y. Israëli, F. Leroux, P. Munsch, J Phys Chem B 2003, $9243-$ 9248.

[53] W. Zhang, J. He, C. Guo, Appl. Clay Sci. 2008, 39, 166-171.

[54] T. Ogasawara, A. Nara, H. Okabayashi, E. Nishio, C. J. O’Connor, Colloid Polym. Sci. 2000, 278, 1070-1084.

[55] J. Li, C. Peng, Z. Li, Z. Wu, S. Li, RSC Adv. 2016, 6, 61393-61401.

[56] J. Wang, L. A. Stevens, T. C. Drage, J. Wood, Chem. Eng. Sci. 2012, 68, 424-431.

[57] S. M. Salili, A. Ataie, M. R. Barati, Z. Sadighi, Mater. Charact. 2015, 106, 78-85. 
[58] G. Socrates, Infrared and Raman Characteristic Group Frequencies: Tables and Charts, Wiley, Chichester, 2010.

[59] Karanveer. S. Aneja, S. Bohm, A. S. Khanna, H. L. M. Bohm, Nanoscale 2015, 7, 1787917888.

[60] R. M. Pasternack, S. Rivillon Amy, Y. J. Chabal, Langmuir 2008, 24, 12963-12971.

[61] F. R. Costa, A. Leuteritz, U. Wagenknecht, D. Jehnichen, L. Häußler, G. Heinrich, Appl. Clay Sci. 2008, 38, 153-164.

[62] J. A. Carrasco, G. Abellán, E. Coronado, J. Mater. Chem. C 2018, 6, 1187-1198.

[63] V. R. Constantino, T. J. Pinnavaia, Inorg. Chem. 1995, 34, 883-892.

[64] S. K. Yun, T. J. Pinnavaia, Chem. Mater. 1995, 7, 348-354.

[65] Z. Qin, P. Yuan, S. Yang, D. Liu, H. He, J. Zhu, Appl. Clay Sci. 2014, 99, 229-236.

[66] T. Xiao, Y. Tang, Z. Jia, D. Li, X. Hu, B. Li, L. Luo, Nanotechnology 2009, 20, 475603.

[67] G. Abellán, E. Coronado, C. J. Gómez-García, C. Martí-Gastaldo, A. Ribera, Polyhedron 2013, 52, 216-221.

[68] G. Abellán, J. L. Jordá, P. Atienzar, M. Varela, M. Jaafar, J. Gómez-Herrero, F. Zamora, A. Ribera, H. García, E. Coronado, Chem Sci 2015, 6, 1949-1958.

[69] M. C. Biesinger, B. P. Payne, A. P. Grosvenor, L. W. M. Lau, A. R. Gerson, R. St. C. Smart, Appl. Surf. Sci. 2011, 257, 2717-2730.

[70] N.-Y. Cui, C. Liu, W. Yang, Surf. Interface Anal. 2011, 43, 1082-1088.

[71] I. George, P. Viel, C. Bureau, J. Suski, G. Lécayon, Surf. Interface Anal. 1996, 24, 774 780.

[72] F. Bauer, H.-J. Gläsel, E. Hartmann, H. Langguth, R. Hinterwaldner, Int. J. Adhes. Adhes. 2004, 24, 519-522.

[73] J. Sun, C. Ou, C. Wang, M. Uchiyama, L. Deng, Organometallics 2015, 34, 1546-1551.

[74] A. Abragam, The Principles of Nuclear Magnetism, Oxford Univ. Press, Oxford, 2007.

[75] Q. Tao, J. Zhu, R. M. Wellard, T. E. Bostrom, R. L. Frost, P. Yuan, H. He, J. Mater. Chem. 2011, 21, 10711-10719.

[76] J. David, Z. Steinmetz, J. Kučerík, G. E. Schaumann, Anal. Chem. 2018, 90, 8793-8799.

[77] E. Conterosito, L. Palin, D. Antonioli, D. Viterbo, E. Mugnaioli, U. Kolb, L. Perioli, M. Milanesio, V. Gianotti, Chem. - Eur. J. 2015, 21, 14975-14986. 
[78] J. Zhang, X. Xie, C. Li, H. Wang, L. Wang, RSC Adv 2015, 5, 29757-29765.

[79] G. Abellán, C. Martí-Gastaldo, A. Ribera, E. Coronado, Acc. Chem. Res. 2015, 48, 16011611.

[80] B. Raveau, M. M. Seikh, Cobalt Oxides: From Crystal Chemistry to Physics, WileyVCH, Weinheim, 2012.

[81] G. Abellán, J. A. Carrasco, E. Coronado, Inorg. Chem. 2013, 52, 7828-7830.

[82] J. J. Almansa, E. Coronado, C. Martí-Gastaldo, A. Ribera, Eur. J. Inorg. Chem. 2008, $2008,5642-5648$.

[83] E. Coronado, J. R. Galán-Mascarós, C. Martí-Gastaldo, A. Ribera, E. Palacios, M. Castro, R. Burriel, Inorg. Chem. 2008, 47, 9103-9110.

[84] K.-H. Goh, T.-T. Lim, Z. Dong, Water Res. 2008, 42, 1343-1368.

[85] L. Bo, Q. Li, Y. Wang, L. Gao, X. Hu, J. Yang, Environ. Prog. Sustain. Energy 2016, DOI 10.1002/ep.12370.

[86] U. Costantino, R. Vivani, M. Bastianini, F. Costantino, M. Nocchetti, Dalton Trans 2014, 43, 11587-11596.

[87] V. Oestreicher, M. Jobbágy, A. E. Regazzoni, Langmuir 2014, 30, 8408-8415.

[88] A. Léonard, R. R. Lauwerys, Mutat. Res. Genet. Toxicol. 1980, 76, 227-239.

[89] G. B. Alexander, W. M. Heston, R. K. Iler, J. Phys. Chem. 1954, 58, 453-455.

[90] J. C. Waerenborgh, P. Salamakha, O. Sologub, A. P. Gonçalves, C. Cardoso, S. Sério, M. Godinho, M. Almeida, Chem. Mater. 2000, 12, 1743-1749.

[91] J. Hesse, A. Rubartsch, J. Phys. [E] 1974, 7, 526-532. 

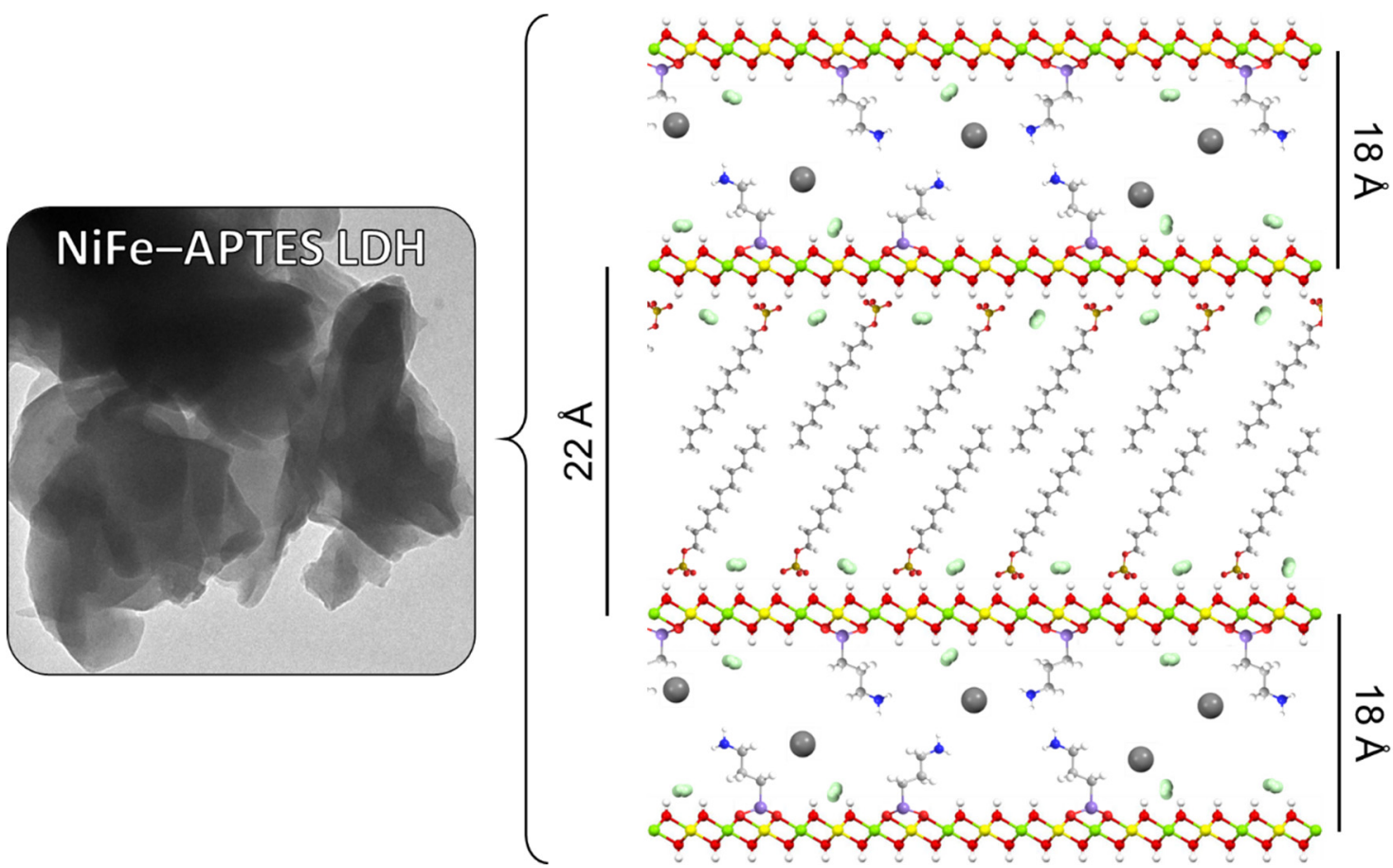

Functionalized NiFe-APTES LDH highlighting the two phases that conforms the second-staging interstratified compound combining the bilayer arrangement of dodecyl sulphate molecules and the (3-aminopropyl)triethoxysilane covalent grafting. 